\title{
ENERGY INEQUALITIES FOR A MODEL OF WAVE PROPAGATION IN COLD PLASMA
}

\author{
THOMAS H. OTWAY
}

\begin{abstract}
Energy inequalities are derived for an elliptic-hyperbolic operator arising in plasma physics. These inequalities imply the existence of distribution and weak solutions to various closed boundary-value problems. An existence theorem is proven for a related class of Keldysh equations, and the failure of expected methods for obtaining uniqueness is discussed. The proofs use ideas recently introduced by Lupo, Morawetz, and Payne for a generalized Tricomi operator. The existence of strong solutions under open boundary conditions is also proven. MSC2000: 35M10, 35D05, 82D10.

Key words: Elliptic-hyperbolic equations, energy inequalities, closed boundary-
\end{abstract} value problems, symmetric-positive operators, equations of Keldysh type

\section{INTRODUCTION}

The equation

$$
\left(x-y^{2}\right) u_{x x}+u_{y y}=0
$$

arises in models of wave propagation through a linear dielectric medium ("cold plasma") at frequencies lying below the geometrical optics range; for the physical context, see 24]. Here $u(x, y),(x, y) \in \mathbb{R}^{2}$, is a scalar function. A subscripted variable denotes partial differentiation in the direction of the variable.

The significant property of eq. (1) is that it changes from elliptic to hyperbolic type along the parabola

$$
x-y^{2}=0 .
$$

By analogy with the equations of steady flow, which change from elliptic to hyperbolic type at the speed of sound, it has become conventional to call this parabola the sonic curve; in the context of the cold plasma model it is also called a resonance curve. Except for a point at the origin, eq. (1) can be mapped into an equation having the same general form as the Tricomi equation

$$
y u_{x x}+u_{y y}=0
$$

an equation which is somewhat more accessible than (11). However, both the physical and mathematical interest of eq. (11) arise from the tangency of the sonic curve to the line $x=0$ at the origin. This is the point at which plasma heating might occur in the physical model, and a point which appears to be singular in numerical studies of solutions; see [17], 22], and [23].

A variety of lower-order terms have been affixed to eq. (1) in the literature; see, e.g., [17, 22, and [25]. The variants reflect different toy models for the equation 
satisfied by the field potential; compare equations (2) and (9) of [22. The variants tend to have the general form

$$
\left(x-y^{2}\right) u_{x x}+u_{y y}+\kappa u_{x}=0,
$$

where the constant $\kappa$ lies in a specified interval. Note that if $\kappa=1$, then the associated differential operator is formally self-adjoint.

The formulation of boundary-value problems for eq. (3) is of considerable interest, as the boundary conditions which are physically natural do not appear to be mathematically natural. In particular, it is shown in [17 that the closed Dirichlet problem, in which the solution is prescribed on the entire boundary, is overdetermined for $C^{2}$ solutions of (3) with $\kappa=1 / 2$. However, the physical properties of electromagnetic waves in the cold plasma model suggest that closed boundaryvalue problems are natural and should be correctly posed for eq. (3).

In Secs. 2 and 3 we prove that solutions to closed boundary-value problems for (31) do in fact exist. Although we do not expect classical solutions, we show in Sec. 2 the existence of distribution solutions to a homogeneous Dirichlet problem, under minimal hypotheses on the domain boundary. In fact these solutions are somewhat smoother than conventional distribution solutions, as they lie in the function space $L^{2}$. An even smoother distribution solution, which lies in a weighted Sobolev space, is derived in Sec. 4 under stronger hypotheses on the boundary. We prove the existence of conventional weak solutions to a class of closed boundaryvalue problems, under strong hypotheses on the boundary, in Sec. 3.

In Sec. 4 we also consider a simpler case in which eq. (11) is replaced by a generalized Cinquini-Cibrario equation [3]

$$
x^{2 k+1} u_{x x}+u_{y y}+\text { lower order }=0,
$$

where $k$ is a non-negative integer. (In fact we do not treat the case $k=0$, which is the case initially studied by Cinquini-Cibrario.) Equation (4) has independent mathematical interest as one of two classes of normal forms to which linear, secondorder elliptic-hyperbolic equations can be reduced near a point 2 . In terms of the physical model, eq. (4) would correspond roughly to a resonance surface which coincides with a flux surface. In this case the plasma behaves like a perpendicular stratified medium and energy absorption occurs along the entire surface, a situation more amenable to standard physical arguments than the case in which the two surfaces are tangent at a single point; see p. 42 of 23 for a discussion.

Equation (4) is an example of an equation of so-called Keldysh type - that is, an equation of the form

$$
K(x) u_{x x}+u_{y y}+\text { lower order }=0,
$$

where $K(x)$ is a continuously differentiable function such that $K(0)=0$ and $x K(x)>0$ for $x \neq 0$. Equations of this kind, with various lower-order terms, arise in transonic fluid dynamics and singular optics (see, e.g., Sec. 3 of [4, and [14]). The arguments of Sec. 4 apply, with only notational alterations, to the slightly more general case of a type-change operator having the form $K(x)=x|x|^{n-1}$ for $n \in \mathbb{R}$, $n>3$. (The proofs require $n$ to be large enough for $K$ to have three continuous derivatives.) 
In Sec. 5 we obtain the existence of unique solutions to eq. (3), but under open boundary conditions, in which the solution is prescribed on a proper subset of the boundary. In Sec. 5.1 we briefly discuss the failure of the preceding methods to provide uniqueness in an obvious way. Conditions for the existence of a unique, strong solution are introduced in Sec. 5.2. The existence of weak $L^{2}$ solutions to a large class of open boundary-value problems is proven in Sec. 6 by extending the arguments of 18 .

The approach taken in Secs. 2-4 is modeled on recent work by Lupo, Morawetz, and Payne [13, 20] on the class of equations

$$
K(y) u_{x x}+u_{y y}=0,
$$

where $K(y)$ is a continuously differentiable function for which $K(0)=0$ and $y K(y)>0$ for $y \neq 0$. In the special case $K(y)=y$, (6) reduces to the Tricomi equation. For this reason, equations of the form (6) are said to be of Tricomi type.

We adapt the ideas of $[13$ to the cold plasma context by proving inequalities for (3) having the form

$$
\left\|L^{*} v\right\|_{U} \geq C\|v\|_{V}
$$

where $C$ is a positive constant, $U$ and $V$ are function spaces, and $L$ is the differential operator of (3) with adjoint $L^{*}$. These energy inequalities are used to show the existence of a solution to boundary-value problems in an appropriate function space (see, e.g., 1, Ch. 2). However, we state the inequalities as theorems, rather than as lemmas, and derive the existence theorems as corollaries. We do this because in their exploitation of inequality (7), the arguments for eqs. (3) and (6) are essentially the same. (While differential operators of Tricomi type are formally self-adjoint, the extension of the existence arguments in [13] to the non-self-adjoint case is standard; see, e.g., the existence proof in 18 for weak solutions to a Dirichlet problem for (3) under open boundary conditions.) The arguments for the two equations differ, however, in their derivations of the energy inequality itself, which depend on the form of the type-change function $K$. One of the main problems of this paper is to find multipliers which allow the Friedrichs $a b c$ method to be applied in the right way, either in its original form or in the more recent integral variant introduced by Didenko [5. Another is to establish a priori restrictions on the domain which allow the method of energy inequalities to be applied. (In Sec. 5.2 we adopt a third approach, also due to Friedrichs, in order to establish sufficient conditions for uniqueness.)

In addition to its physical interest, the existence of solutions to closed boundaryvalue problems for equations of the form (3) and (4) has purely mathematical interest as an extension of the methods introduced in 13 to equations which are not of Tricomi type. The existence of solutions to closed boundary-value problems for certain elliptic-hyperbolic systems which are not of Tricomi type is shown in 7 and 14 under special conditions on the boundary that do not require the methods of [13]. Our results suggest that the existence - but not necessarily uniqueness of distribution and weak solutions for equations of Keldysh type can be shown by arguments closely modeled on those of [13].

The following hypotheses on the domain $\Omega$ are assumed throughout: It is a bounded, connected domain of $\mathbb{R}^{2}$ having piecewise smooth boundary $\partial \Omega$, oriented 
in a counterclockwise direction; the domain includes both an arc of the sonic curve and the origin of coordinates in $\mathbb{R}^{2}$ (so that, for example, eq. (3) is elliptic-hyperbolic but not equivalent to an equation of Tricomi type). We neither require nor exclude the existence of characteristic arcs on the boundary. We use the term elliptic boundary to refer to points $(x, y)$ of the domain boundary on which the typechange function $K(x, y)$ is positive. Similarly, by the hyperbolic boundary we mean boundary points for which the type-change function is negative.

\section{INEQUALITIES LEADING TO DISTRIBUTION SOLUTIONS}

The function spaces introduced in [5] and 13 reappear in this paper with $|K(y)|$ replaced by a different weight function, also denoted by $K$. In particular, we define the space $H_{0}^{1}(\Omega ; K)$ to be the closure of $C_{0}^{\infty}(\Omega)$ with respect to the norm

$$
\|u\|_{H^{1}(\Omega ; K)}=\left[\iint_{\Omega}\left(|K| u_{x}^{2}+u_{y}^{2}+u^{2}\right) d x d y\right]^{1 / 2},
$$

where $|K|=\left|x-y^{2}\right|$. We can write the $H_{0}^{1}(\Omega ; K)$-norm in the form

$$
\|u\|_{H_{0}^{1}(\Omega ; K)}=\left[\iint_{\Omega}\left(|K| u_{x}^{2}+u_{y}^{2}\right) d x d y\right]^{1 / 2}
$$

as a consequence of the weighted Poincaré inequality

$$
\|u\|_{L^{2}(\Omega)}^{2} \leq C \iint_{\Omega}\left(|K| u_{x}^{2}+u_{y}^{2}\right) d x d y .
$$

Here and below we denote by $C$ generic positive constants, the value of which may change from line to line.

The complexity of the existence arguments is not increased if we replace (3) by the inhomogeneous equation

$$
L u=f,
$$

where $f$ is a given, sufficiently smooth function of $(x, y)$ and

$$
L=\left(x-y^{2}\right) \frac{\partial^{2}}{\partial x^{2}}+\frac{\partial^{2}}{\partial y^{2}}+\kappa \frac{\partial}{\partial x} .
$$

By a distribution solution of equations (9), (10) with the boundary condition

$$
u(x, y)=0 \forall(x, y) \in \partial \Omega
$$

we mean a function $u \in L^{2}(\Omega)$ such that $\forall \xi \in H_{0}^{1}(\Omega ; K)$ for which $L^{*} \xi \in L^{2}(\Omega)$, we have

$$
\left(u, L^{*} \xi\right)=\langle f, \xi\rangle
$$

Here (, ) denotes the $L^{2}$ inner product on $\Omega$ and $\langle$,$\rangle is the duality bracket associated$ to the $H^{-1}$ norm 8

$$
\|w\|_{H^{-1}(\Omega ; K)}=\sup _{0 \neq \xi \in C_{0}^{\infty}(\Omega)} \frac{|\langle w, \xi\rangle|}{\|\xi\|_{H_{0}^{1}(\Omega ; K)}} .
$$


Such a solution is a little smoother than the usual notion of distribution solution, in which the solution fails to lie in a true function space.

Theorem 1. Every $u \in C_{0}^{2}(\Omega)$ satisfies the inequality

$$
\|u\|_{H_{0}^{1}(\Omega ; K)} \leq C|| L u \|_{L^{2}(\Omega)}
$$

where $L$ is defined by (10) with $\kappa \in[0,2]$, and $K=x-y^{2}$.

Proof. We consider two cases.

Case 1: $1 \leq \kappa \leq 2$. Let $\delta$ be a small, positive constant. Define an operator $M$ by the identity

$$
M u=a u+b u_{x}+c u_{y}
$$

for $a=-1, c=2(2 \delta-1) y$, and

$$
b=\left\{\begin{array}{ll}
\exp \left(2 \delta K / Q_{1}\right) & \text { if }(x, y) \in \Omega^{+} \\
\exp \left(6 \delta K / Q_{2}\right) & \text { if }(x, y) \in \Omega^{-}
\end{array},\right.
$$

where

$$
\Omega^{+}=\{(x, y) \in \Omega \mid K>0\}
$$

and $\Omega^{-}=\Omega \backslash \Omega^{+}$. Choose $Q_{1}=\exp \left(2 \delta \mu_{1}\right)$, where

$$
\mu_{1}=\max _{(x, y) \in \overline{\Omega^{+}}} K
$$

Then $\forall(x, y) \in \Omega^{+}$, we have

$$
2 \delta K \leq 2 \delta \mu_{1} \leq 2 \delta \mu_{1} e^{2 \delta \mu_{1}}=Q_{1} \log Q_{1} .
$$

Dividing by $Q_{1}$ and exponentiating both sides, we conclude that $b \leq Q_{1}$ on $\Omega^{+}$. Define the negative number $\mu_{2}$ by

$$
\mu_{2}=\min _{(x, y) \in \overline{\Omega^{-}}} K
$$

and let $Q_{2}=\exp \left(\mu_{2}\right)$. Then $0<Q_{2}<1$ and, for given $\Omega$, we can choose $\delta$ to be so small that $6 \delta<Q_{2}$. In that case, $\forall(x, y) \in \Omega^{-}$,

$$
6 \delta K \geq 6 \delta \mu_{2}=6 \delta \log Q_{2}>Q_{2} \log Q_{2} .
$$

We conclude that $b>Q_{2}$ on $\Omega^{-}$.

We will estimate the quantity $(M u, L u)$ from above and below. Integrating by parts, we have

$$
(M u, L u)=\iint_{\Omega} \sum_{i=1}^{9} \tau_{i} d x d y
$$

where

$$
\begin{gathered}
\tau_{1}=\left(a u K u_{x}\right)_{x}-a_{x} K u u_{x}-\frac{1}{2}\left(a u^{2}\right)_{x}+\frac{a_{x}}{2} u^{2}-a K u_{x}^{2} \\
\tau_{2}=\left(a u u_{y}\right)_{y}-a_{y} u u_{y}-a u_{y}^{2}
\end{gathered}
$$




$$
\begin{gathered}
\tau_{3}=\frac{1}{2}\left(b K u_{x}^{2}\right)_{x}-\frac{1}{2}\left(b_{x} K+b\right) u_{x}^{2} ; \\
\tau_{4}=\left(b u_{x} u_{y}\right)_{y}-b_{y} u_{x} u_{y}-\frac{1}{2}\left(b u_{y}^{2}\right)_{x}+\frac{b_{x}}{2} u_{y}^{2} ; \\
\tau_{5}=\left(c u_{y} K u_{x}\right)_{x}-c_{x} K u_{x} u_{y} \\
-\frac{1}{2}\left(c K u_{x}^{2}\right)_{y}-c u_{x} u_{y}+\left(\frac{c_{y}}{2} K-c y\right) u_{x}^{2} ; \\
\tau_{6}=\frac{1}{2}\left[\left(c u_{y}^{2}\right)_{y}-c_{y} u_{y}^{2}\right]
\end{gathered}
$$

the lower-order terms are:

$$
\begin{gathered}
\tau_{7}=\frac{\kappa}{2}\left[\left(a u^{2}\right)_{x}-\kappa a_{x} u^{2}\right] ; \\
\tau_{8}=\kappa b u_{x}^{2} ; \\
\tau_{9}=\kappa c u_{y} u_{x} .
\end{gathered}
$$

As in the Tricomi case considered in [13, one of the coefficients in $M u$ fails to be continuously differentiable on all of $\Omega$. When integrating this quantity, a cut should be introduced along the line $K=0$ separating $\Omega^{+}$from $\Omega^{-}$. The boundary integrals involving $a, b$, and $c$ on either side of this line will cancel by continuity.

We find that the boundary terms vanish by the compact support of $u$, and

where $\omega=0$;

$$
(M u, L u)=\iint_{\Omega^{+} \cup \Omega^{-}} \omega u^{2}+\alpha u_{x}^{2}+2 \beta u_{x} u_{y}+\gamma u_{y}^{2} d x d y,
$$

for

$$
\alpha=\left(\frac{c_{y}}{2}-a-\frac{b_{x}}{2}\right) K+\left(\kappa-\frac{1}{2}\right) b-c y
$$

and

$$
\alpha_{\mid \Omega^{+}}=\left(2-\frac{b}{Q_{1}}\right) \delta K+2(1-2 \delta) y^{2}+\left(\kappa-\frac{1}{2}\right) b
$$

$$
\begin{gathered}
\alpha_{\mid \Omega^{-}}=\left(3 \frac{b}{Q_{2}}-2\right) \delta|K|+2(1-2 \delta) y^{2}+\left(\kappa-\frac{1}{2}\right) b ; \\
\beta=\frac{1}{2}\left[c(\kappa-1)-b_{y}\right]=\left\{\begin{array}{cl}
y\left[2 \delta\left(b / Q_{1}\right)+(\kappa-1)(2 \delta-1)\right] \leq|y| & \text { in } \Omega^{+} \\
y\left[6 \delta\left(b / Q_{2}\right)+(\kappa-1)(2 \delta-1)\right] \leq \kappa|y| & \text { in } \Omega^{-}
\end{array} ;\right. \\
\gamma=\frac{1}{2}\left(b_{x}-c_{y}\right)-a=\left\{\begin{array}{cl}
2(1-\delta)+\delta\left(b / Q_{1}\right) & \text { in } \Omega^{+} \\
2(1-\delta)+3 \delta\left(b / Q_{2}\right) & \text { in } \Omega^{-}
\end{array}\right.
\end{gathered}
$$

On $\Omega^{+}$, for any scalars $\xi$ and $\eta$, we have by Cauchy's inequality

$$
2 \beta \xi \eta \leq y^{2} \xi^{2}+\eta^{2},
$$

so

$$
\begin{gathered}
\alpha \xi^{2}+2 \beta \xi \eta+\gamma \eta^{2} \geq \alpha \xi^{2}-\left(y^{2} \xi^{2}+\eta^{2}\right)+\gamma \eta^{2}= \\
{\left[\left(2-\frac{b}{Q_{1}}\right) \delta K+(1-4 \delta) y^{2}+\left(\kappa-\frac{1}{2}\right) b\right] \xi^{2}+\left[(1-2 \delta)+\frac{6 b}{Q_{1}}\right] \eta^{2}}
\end{gathered}
$$




$$
\geq \delta\left(K \xi^{2}+\eta^{2}\right)
$$

provided $\delta$ is sufficiently small. On $\Omega^{-}$,

$$
2 \beta \xi \eta \leq 2|\kappa y \xi \eta| \leq 2\left(y^{2} \xi^{2}+\eta^{2}\right),
$$

so

$$
\begin{gathered}
\alpha \xi^{2}+2 \beta \xi \eta+\gamma \eta^{2} \geq \alpha^{2} \xi^{2}-2\left(y^{2} \xi^{2}+\eta^{2}\right)+\gamma \eta^{2}= \\
{\left[\left(3 \frac{b}{Q_{2}}-2\right) \delta|K|-4 \delta y^{2}+\left(\kappa-\frac{1}{2}\right) b\right] \xi^{2}+\delta\left(3 \frac{b}{Q_{2}}-2\right) \eta^{2}} \\
\geq \delta\left(|K| \xi^{2}+\eta^{2}\right) .
\end{gathered}
$$

In estimating the coefficient of $\eta^{2}$, we used the fact that $b>Q_{2}$ on $\Omega^{-}$. In estimating the coefficient of $\xi^{2}$, we used the fact that

$$
\left(\kappa-\frac{1}{2}\right) b-4 \delta y^{2} \geq \frac{b}{2}-4 \delta y^{2} \geq \frac{1}{2} e^{\mu_{2}}-4 \delta y^{2},
$$

which exceeds zero for $\delta$ sufficiently small with respect to $\exp \left(-\left|\mu_{2}\right|\right)$ and $\max _{\bar{\Omega}} y^{2}$.

Integrating over each subdomain, we obtain

$$
(M u, L u) \geq \delta \iint_{\Omega}\left(|K| u_{x}^{2}+u_{y}^{2}\right) d x d y=\delta\|u\|_{H_{0}^{1}(\Omega ; K)}^{2} .
$$

We want to obtain an upper bound for $(M u, L u)$ in terms of the $L^{2}$-norm of $L u$. First we recall that $\max _{(x, y) \in \Omega} b \leq Q_{1}$ and estimate

$$
(M u, L u) \leq \iint_{\Omega}|u||L u| d x d y+
$$

$$
C \iint_{\Omega}\left(Q_{1}\left|u_{x}\right|+|y|\left|u_{y}\right|\right)|L u| d x d y=i_{1}+i_{2} .
$$

The Schwarz inequality and inequality (8) imply that

Similarly,

$$
i_{1} \leq\|u\|_{L^{2}(\Omega)}\|L u\|_{L^{2}(\Omega)} \leq C\|u\|_{H_{0}^{1}(\Omega ; K)}\|L u\|_{L^{2}(\Omega)} .
$$

$$
\begin{gathered}
i_{2} \leq C\left[\iint_{\Omega}\left(\left|u_{x}\right|+\left|u_{y}\right|\right)^{2} d x d y\right]^{1 / 2}\|L u\|_{L^{2}(\Omega)} \\
\leq C^{\prime}\|u\|_{H_{0}^{1}(\Omega)}\|L u\|_{L^{2}(\Omega)} .
\end{gathered}
$$

We claim that we can choose $\delta$ to be so small that $b / 2 \geq \delta$ on $\Omega$. On $\Omega^{+}$this is obvious, as $b \geq 1$ there. On $\Omega^{-}$it is almost as obvious, as $b>Q_{2}>6 \delta>2 \delta$. Thus we have the additional estimate

implying that

$$
\alpha \geq \delta|K|+\frac{b}{2} \geq \delta
$$




$$
(M u, L u) \geq \delta\|u\|_{H_{0}^{1}(\Omega)}^{2} .
$$

Substituting the estimates for $i_{1}$ and $i_{2}$ into inequality (14) and combining this with (15), we obtain

$$
\begin{gathered}
\delta\|u\|_{H_{0}^{1}(\Omega)}^{2} \leq(M u, L u) \leq \\
C\left(\|u\|_{H_{0}^{1}(\Omega ; K)}+\|u\|_{H_{0}^{1}(\Omega)}\right)\|L u\|_{L^{2}(\Omega)} \leq C^{\prime}\|u\|_{H_{0}^{1}(\Omega)}\|L u\|_{L^{2}(\Omega)} .
\end{gathered}
$$

Dividing eq. (16) by the $H_{0}^{1}$-norm of $u$, we find that

$$
\|u\|_{H_{0}^{1}(\Omega)} \leq C|| L u \|_{L^{2}(\Omega)} .
$$

This completes the proof for the case $\kappa \in[1,2]$, as the norm on the left can be replaced by the corresponding weighted norm. (Alternatively, substitute this estimate into the extreme right-hand side of (16) and replace the extreme left-hand side of (16) by the extreme right-hand side of (13), to obtain

$$
\|u\|_{H_{0}^{1}(\Omega ; K)}^{2} \leq C|| L u \|_{L^{2}(\Omega)}^{2}
$$

then take the square root of each side.)

Case 2: $0 \leq \kappa<1$. Again subdivide the domain into $\Omega^{+}$and $\Omega^{-}$by introducing a cut along the curve $K=0$. Integrate by parts as in case 1 , choosing $a=-1$;

$$
b=\left\{\begin{array}{cl}
-N K & \text { in } \Omega^{+} \\
N K & \text { in } \Omega^{-}
\end{array},\right.
$$

where $N$ is a constant satisfying

$$
\frac{1+\tilde{\delta}}{3-\kappa}<N<\frac{1-\tilde{\delta}}{\kappa+1}
$$

for a sufficiently small positive constant $\tilde{\delta}$, and

$$
c=-4 N y \text {. }
$$

The boundary integrals involving $a$ and $c$ on either side of the curve will cancel and the boundary integrals involving $b$ will be zero on the curve.

On $\Omega^{+}$,

$$
\begin{gathered}
\alpha=[1-(1+\kappa) N] K+4 N y^{2} ; \\
\beta=N(1-2 \kappa) y ; \\
\gamma=1+\frac{3}{2} N .
\end{gathered}
$$

Also,

as $\kappa \in[0,1)$, so

$$
2 \beta \xi \eta \geq-2 N|y \xi \eta| \geq-N\left(y^{2} \xi^{2}+\eta^{2}\right)
$$




$$
\begin{gathered}
\alpha \xi^{2}+2 \beta \xi \eta+\gamma \eta^{2} \geq \alpha \xi^{2}-N\left(y^{2} \xi^{2}+\eta^{2}\right)+\gamma \eta^{2}= \\
\left\{[1-(1+\kappa) N] K+3 N y^{2}\right\} \xi^{2}+\left(1+\frac{N}{2}\right) \eta^{2} \geq \tilde{\delta}\left(K \xi^{2}+\eta^{2}\right),
\end{gathered}
$$

by condition (17).

On $\Omega^{-}$,

$$
\begin{gathered}
\alpha=-[1-(3-\kappa) N]|K|+4 N y^{2} ; \\
\beta=N(3-2 \kappa) y \\
\gamma=1+\frac{5}{2} N .
\end{gathered}
$$

Because

we have

$$
2 \beta \xi \eta \geq-6 N|y \xi \eta|
$$

$$
\begin{gathered}
\alpha \xi^{2}+2 \beta \xi \eta+\gamma \eta^{2} \geq \\
\left\{-[1-(3-\kappa) N]|K|+4 N y^{2}\right\} \xi^{2}-3 N\left(y^{2} \xi^{2}+\eta^{2}\right)+\left(1+\frac{5}{2} N\right) \eta^{2}= \\
\left\{-[1-(3-\kappa) N]|K|+N y^{2}\right\} \xi^{2}+\left(1-\frac{N}{2}\right) \eta^{2} \geq \tilde{\delta}\left(|K| \xi^{2}+\eta^{2}\right),
\end{gathered}
$$

again using (17).

We conclude that

$$
(M u, L u) \geq \tilde{\delta}\|u\|_{H_{0}^{1}(\Omega ; K)}^{2} .
$$

In order to obtain an upper bound for $(M u, L u)$ in terms of the $L^{2}$-norm of $L u$, we estimate

$$
\begin{gathered}
(M u, L u) \leq \iint_{\Omega}\left\{|u|+N\left[|K|\left|u_{x}\right|+4|y|\left|u_{y}\right|\right]\right\}|L u| d x d y \\
=\iint_{\Omega}|u||L u| d x d y+N \iint_{\Omega}\left[|K|\left|u_{x}\right|+4|y|\left|u_{y}\right|\right]|L u| d x d y \\
\equiv i_{1}+i_{2} .
\end{gathered}
$$

As in case 1, the Schwarz inequality and (8) imply that

$$
i_{1} \leq\|u\|_{L^{2}(\Omega)}\|L u\|_{L^{2}(\Omega)} \leq C\|u\|_{H_{0}^{1}(\Omega ; K)}\|L u\|_{L^{2}(\Omega)} .
$$

Because $\Omega$ is bounded, we can fit it inside a rectangle of the form

$$
R=\left\{(x, y) \mid \gamma_{R} \leq x \leq \delta_{R}, \beta_{R} \leq y \leq \alpha_{R}\right\} .
$$

for sufficiently large values of $\left|\alpha_{R}\right|,\left|\beta_{R}\right|,\left|\gamma_{R}\right|$, and $\left|\delta_{R}\right|$. Define 


$$
T=\max \left\{\left|\delta_{R}\right|+\alpha_{R}^{2}, 1\right\}
$$

Then

$$
\begin{gathered}
i_{2} \leq N \iint_{\Omega}\left[\sqrt{T} \sqrt{|K|}\left|u_{x}\right|+4|y|\left|u_{y}\right|\right]|L u| d x d y \\
\leq N\left[\iint_{\Omega} \Upsilon d x d y\right]^{1 / 2}\|L u\|_{L^{2}(\Omega)},
\end{gathered}
$$

where

$$
\Upsilon=T|K|\left|u_{x}\right|^{2}+8 \sqrt{T}\left|\alpha_{R}\right| \sqrt{|K|}\left|u_{x}\right|\left|u_{y}\right|+16 \alpha_{R}^{2}\left|u_{y}\right|^{2} .
$$

Applying Cauchy's inequality to $\Upsilon$, we obtain

$$
i_{2} \leq C\|u\|_{H_{0}^{1}(\Omega ; K)}\|L u\|_{L^{2}(\Omega)},
$$

where $C$ depends on $N$ and $R$.

Substituting the estimates for $i_{1}$ and $i_{2}$ into inequality (19), we obtain the desired upper bound for $(M u, L u)$. Combining this with the lower bound (18) and dividing through by the $H_{0}^{1}(\Omega ; K)$-norm of $u$, we complete the proof of Theorem 1 .

Corollary 2. The Dirichlet problem (9), (10), (11) with $\kappa \in[0,2]$ possesses a distribution solution $u \in L^{2}(\Omega)$ for every $f \in H^{-1}(\Omega ; K)$.

Proof. The proof for the case $\kappa=1$ is identical to the proof of Theorem 2.2 of 13, with Lemma 2.1 of 13 replaced by Theorem 1 of the present communication. Briefly, we define for $\xi \in C_{0}^{\infty}$ a linear functional

$$
J_{f}(L \xi)=\langle f, \xi\rangle .
$$

This functional is bounded on a subspace of $L^{2}$ by the inequality

$$
|\langle f, \xi\rangle| \leq\|f\|_{H^{-1}(\Omega ; K)}\|\xi\|_{H_{0}^{1}(\Omega ; K)}
$$

and by Theorem 1 (applied to the second term on the right). Now standard HahnBanach arguments extend the functional to one defined on all of $L^{2}$. The Riesz Representation Theorem then guarantees the existence of the distribution solution.

If $\kappa \neq 1$, then $L$ is not self-adjoint. Because the operator adjoint to $L$ has the form

$$
L^{*}=\left(x-y^{2}\right) \frac{\partial^{2}}{\partial x^{2}}+\frac{\partial^{2}}{\partial y^{2}}+(2-\kappa) \frac{\partial}{\partial x},
$$

estimating $L$ for $\kappa$ in $[0,2]$ will also yield estimates for $L^{*}$. Applying the preceding argument to the adjoint operator completes the proof of Corollary 2. 


\section{INEQUALITIES LEADING TO WEAK SOLUTIONS}

Applications to plasma physics of mixed boundary-value problems are discussed in [9]. But we adopt mixed boundary conditions in this section mostly in the interest of mathematical generality. The main interest for these equations is in closed Dirichlet and Neumann problems. The arguments of this section will extend the results of [18, for an open weak Dirichlet problem for eq. (1), to a class of generalized, closed Neumann problems.

If $\mathbf{u}=\left(u_{1}, u_{2}\right)$ and $\mathbf{w}=\left(w_{1}, w_{2}\right)$ are measurable vector-valued functions on $\Omega$, then the inner product for the space $L^{2}\left(\Omega ; \mathbb{R}^{2}\right)$ will be written

$$
(\mathbf{u}, \mathbf{w})_{L^{2}\left(\Omega ; \mathbb{R}^{2}\right)}=\iint_{\Omega}\left(u_{1} w_{1}+u_{2} w_{2}\right) d x d y .
$$

Analogously, we define $\mathcal{H}_{\mathcal{K}}$ to be the Hilbert space of measurable functions on $\Omega$ for which the weighted $L^{2}$-norm

$$
\|\mathbf{u}\|_{H_{\mathcal{K}}}=\left[\iint_{\Omega}\left(|K| u_{1}^{2}+u_{2}^{2}\right) d x d y\right]^{1 / 2}
$$

is finite; this norm is induced by the the weighted $L^{2}$ inner product

$$
(\mathbf{u}, \mathbf{w})_{\mathcal{K}}=\iint_{\Omega}\left(|K| u_{1} w_{1}+u_{2} w_{2}\right) d x d y .
$$

In the notation for these spaces, $\mathcal{K}$ denotes a diagonal matrix having entries $|K|$ and 1.

By a weak solution of a mixed boundary-value problem in this context we mean an element $\mathbf{u} \in \mathcal{H}_{\mathcal{K}}(\Omega)$ such that

$$
-\left(\mathbf{u}, \mathcal{L}^{*} \mathbf{w}\right)_{L^{2}\left(\Omega ; \mathbb{R}^{2}\right)}=(\mathbf{f}, \mathbf{w})_{L^{2}\left(\Omega ; \mathbb{R}^{2}\right)}
$$

for every function $\mathbf{w} \in C^{1}\left(\bar{\Omega} ; \mathbb{R}^{2}\right)$ for which $\mathcal{K}^{-1} \mathcal{L}^{*} \mathbf{w} \in L^{2}\left(\Omega ; \mathbb{R}^{2}\right)$ and for which

$$
w_{1}=0 \forall(x, y) \in G
$$

and

$$
w_{2}=0 \forall(x, y) \in \partial \Omega \backslash G,
$$

where $G$ is a (possibly empty) subset of $\partial \Omega$. With a view toward providing a firstorder generalization of eq. (3), we choose the differential operator $\mathcal{L}$ to have the form

$$
\left(\begin{array}{cc}
K \partial_{x} & \partial_{y} \\
\partial_{y} & -\partial_{x}
\end{array}\right)+\left(\begin{array}{cc}
\kappa & 0 \\
0 & 0
\end{array}\right)
$$

Theorem 3. Let $G$ be a subset of $\partial \Omega$ and let $K=x-y^{2}$. Define the functions $b(x, y)=m K+s$ and $c(y)=\mu y-t$, where $\mu$ is a positive constant,

$$
m= \begin{cases}(\mu+\delta) / 2 & \text { in } \Omega^{+} \\ (\mu-\delta) / 2 & \text { in } \Omega^{-}\end{cases}
$$


for a small positive number $\delta$, and $t$ is a positive constant such that $\mu y-t<0 \forall y \in$ $\Omega$. Let $s$ be a sufficiently large positive constant. In particular, choose $s$ to be so large that the quantities $m K+s, 2 c y+s$, and $b^{2}+K c^{2}$ are all positive. Let

$$
b d y-c d x \leq 0
$$

on $G$ and

$$
K(b d y-c d x) \geq 0
$$

on $\partial \Omega \backslash G$. Then there exists a positive constant $C$ such that

$$
\left(\boldsymbol{\Psi}, \mathcal{L}^{*} \mathcal{M} \Psi\right) \geq C \iint_{\Omega}\left(|K| \Psi_{1}^{2}+\Psi_{2}^{2}\right) d x d y
$$

for any sufficiently smooth 2-vector $\boldsymbol{\Psi}$, provided conditions (24), (25) are satisfied on the boundary for $\mathbf{w}=\mathcal{M} \boldsymbol{\Psi}$, where $\mathcal{L}^{*}$ is given by (26) with $\kappa=1$ and

$$
\mathcal{M}=\left(\begin{array}{cc}
b & c \\
-K c & b
\end{array}\right) .
$$

Proof. Again the proof closely follows 13 (Lemmas 4.2 and 4.3, and Theorem 4.4). After integration by parts and an application of the Divergence Theorem, we find that

$$
\begin{gathered}
\left(\Psi, \mathcal{L}^{*} \mathcal{M}\right)_{L^{2}\left(\Omega ; \mathbb{R}^{2}\right)}=\iint_{\Omega}\left(\alpha \Psi_{1}^{2}+2 \beta \Psi_{1} \Psi_{2}+\gamma \Psi_{2}^{2}\right) d x d y+ \\
\int_{\partial \Omega}\left(\frac{1}{2} K c \Psi_{1}^{2}-b \Psi_{1} \Psi_{2}-\frac{c}{2} \Psi_{2}^{2}\right) d x+\left(\frac{1}{2} K b \Psi_{1}^{2}+K c \Psi_{1} \Psi_{2}-\frac{1}{2} b \Psi_{2}^{2}\right) d y
\end{gathered}
$$

Because $b$ is not continuously differentiable on $\Omega$, it is again necessary to introduce a cut along the resonance curve $x=y^{2}$. Evaluating the line integral in (29) for our choices of $b$ and $c$, using the fact that $c$ is continuous and that the discontinuous term in $b$ vanishes on the resonance curve, we find that the boundary integrals sum to zero along the cut. Applying the boundary conditions, we obtain

$$
\begin{aligned}
\int_{\partial \Omega}\left(\frac{1}{2} K c \Psi_{1}^{2}-\right. & \left.b \Psi_{1} \Psi_{2}-\frac{c}{2} \Psi_{2}^{2}\right) d x+\left(\frac{1}{2} K b \Psi_{1}^{2}+K c \Psi_{1} \Psi_{2}-\frac{1}{2} b \Psi_{2}^{2}\right) d y \\
= & \frac{1}{2} \int_{G} \frac{-1}{c^{2}} \Psi_{1}^{2}\left(b^{2}+K c^{2}\right)(b d y-c d x) \\
+ & \frac{1}{2} \int_{\partial \Omega \backslash G} \frac{1}{b^{2}} \Psi_{1}^{2}\left(b^{2}+K c^{2}\right) K(b d y-c d x) .
\end{aligned}
$$

The hypotheses insure that the line integrals in (30) are finite and nonnegative. We have

$$
\alpha=\frac{1}{2}\left[K b_{x}-K_{x} b-(K c)_{y}\right]+b=(\delta / 2)\left|x-y^{2}\right|+y(\mu y-t)+s / 2
$$


Because $\forall \varepsilon>0$

$$
\begin{gathered}
\beta=\frac{1}{2}\left(b_{y}+c\right)=\left\{\begin{array}{cc}
-(1 / 2)(\delta y+t) & \text { in } \Omega^{+} \\
(1 / 2)(\delta y-t) & \text { in } \Omega^{-}
\end{array} ;\right. \\
\gamma=\frac{1}{2}\left(c_{y}-b_{x}\right)=\left\{\begin{array}{cc}
(\mu-\delta) / 4 & \text { in } \Omega^{+} \\
(\mu+\delta) / 4 & \text { in } \Omega^{-}
\end{array}\right.
\end{gathered}
$$

it follows that

$$
2 \beta \Psi_{1} \Psi_{2} \geq-\frac{(|\delta y|+|t|)^{2}}{\varepsilon} \Psi_{1}^{2}-\varepsilon \Psi_{2}^{2},
$$

$$
\begin{gathered}
\iint_{\Omega}\left(\alpha \Psi_{1}^{2}+2 \beta \Psi_{1} \Psi_{2}+\gamma \Psi_{2}^{2}\right) d x d y \geq \\
\iint_{\Omega}\left\{\left[\frac{\delta}{2}|K|+y(\mu y-t)+\frac{s}{2}-\frac{(|\delta y|+|t|)^{2}}{\varepsilon}\right] \Psi_{1}^{2}+\left[\frac{(\mu-\delta)}{4}-\varepsilon\right] \Psi_{2}^{2}\right\} d x d y \\
\geq C \iint_{\Omega}\left(|K| \Psi_{1}^{2}+\Psi_{2}^{2}\right) d x d y
\end{gathered}
$$

for $s$ sufficiently large, $\varepsilon$ sufficiently small, and $\delta<\mu$. This completes the proof.

Define the space $\mathcal{H}_{\mathcal{K}^{-1}}(\Omega)$ to consist of measurable functions $\mathbf{f}=\left(f_{1}, f_{2}\right)$ on $\Omega$ for which $\mathcal{K}^{-1} \mathbf{f}$ lies in the space $L^{2}\left(\Omega ; \mathbb{R}^{2}\right)$, where

$$
\mathcal{K}^{-1}=\left(\begin{array}{cc}
|K|^{-1} & 0 \\
0 & 1
\end{array}\right) .
$$

Then $\mathcal{H}_{\mathcal{K}^{-1}}(\Omega)$ is a Hilbert space having inner product

$$
(\mathbf{v}, \mathbf{w})_{\mathcal{H}_{\mathcal{K}^{-1}}(\Omega)}=\left(\mathcal{K}^{-2} \mathbf{v}, \mathbf{w}\right)_{L^{2}\left(\Omega ; \mathbb{R}^{2}\right)} .
$$

Corollary 4. Under the hypotheses of Theorem 3, there exists for every $\mathbf{f}$ such that $\mathcal{K}^{-1} \mathcal{M}^{T} \mathbf{f} \in L^{2}(\Omega)$ a weak solution to the mixed boundary-value problem (23)(25) with $\mathcal{L}$ given by eq. (26) with $\kappa=0$. (The superscripted $T$ denotes matrix transpose.)

Proof. Apply the proof of Theorem 3 in [16] (c.f. [13], Lemmas 4.2 and 4.3, and 21]), taking into account that in our case, $\mathcal{L} \neq \mathcal{L}^{*}$. Use Theorem 3 of this paper to estimate the formal adjoint of $\mathcal{L}$, which is obtained by taking $\kappa=1$ in (26). Because $\mathcal{M} \Psi=\mathbf{w}$, we have

$$
\begin{gathered}
\iint_{\Omega}\left(|K| \Psi_{1}^{2}+\Psi_{2}^{2}\right) d x d y=\|\Psi\|_{\mathcal{H}_{\mathcal{K}}(\Omega)} \\
=\left\|\mathcal{M}^{-1} \mathbf{w}\right\|_{\mathcal{H}_{\mathcal{K}}(\Omega)}=\left\|\mathcal{K} \mathcal{M}^{-1} \mathbf{w}\right\|_{L^{2}\left(\Omega ; \mathbb{R}^{2}\right)}
\end{gathered}
$$

for

and

$$
\mathcal{K}=\left(\begin{array}{cc}
|K| & 0 \\
0 & 1
\end{array}\right)
$$




$$
\mathcal{M}^{-1} \mathbf{w}=\frac{1}{b^{2}+K c^{2}}\left(\begin{array}{c}
b w_{1}-c w_{2} \\
c K w_{1}+b w_{2}
\end{array}\right)
$$

These norms are finite because of the hypotheses on $\mathbf{w}$ and on $b^{2}+K c^{2}$. Using Theorem 3, we obtain

$$
\begin{aligned}
& \frac{\delta}{2}\left\|\mathcal{K} \mathcal{M}^{-1} \mathbf{w}\right\|_{L^{2}\left(\Omega ; \mathbb{R}^{2}\right)}^{2} \leq\left(\boldsymbol{\Psi}, \mathcal{L}^{*} \mathcal{M} \Psi\right)_{L^{2}\left(\Omega ; \mathbb{R}^{2}\right)}=\left(\mathcal{M}^{-1} \mathbf{w}, \mathcal{L}^{*} \mathbf{w}\right)_{L^{2}\left(\Omega ; \mathbb{R}^{2}\right)} \\
= & \left(\mathcal{K} \mathcal{M}^{-1} \mathbf{w}, \mathcal{K}^{-1} \mathcal{L}^{*} \mathbf{w}\right)_{L^{2}\left(\Omega ; \mathbb{R}^{2}\right)} \leq\left\|\mathcal{K} \mathcal{M}^{-1} \mathbf{w}\right\|_{L^{2}\left(\Omega ; \mathbb{R}^{2}\right)}\left\|\mathcal{K}^{-1} \mathcal{L}^{*} \mathbf{w}\right\|_{L^{2}\left(\Omega ; \mathbb{R}^{2}\right)},
\end{aligned}
$$

in which the right-hand side is finite by our definition of $\mathbf{w}$. Dividing both sides of this inequality by the quantity $\left\|\mathcal{K} \mathcal{M}^{-1} \mathbf{w}\right\|_{L^{2}\left(\Omega ; \mathbb{R}^{2}\right)}$, we find that

$$
\left\|\mathcal{K} \mathcal{M}^{-1} \mathbf{w}\right\|_{L^{2}\left(\Omega ; \mathbb{R}^{2}\right)} \leq \frac{2}{\delta}\left\|\mathcal{K}^{-1} \mathcal{L}^{*} \mathbf{w}\right\|_{L^{2}\left(\Omega ; \mathbb{R}^{2}\right)} .
$$

Define the linear functional

$$
J_{f}\left(\mathcal{L}^{*} \mathbf{w}\right)=(\mathbf{f}, \mathbf{w})_{L^{2}\left(\Omega ; \mathbb{R}^{2}\right)}
$$

Then

$$
\left|J_{f}\left(\mathcal{L}^{*} \mathbf{w}\right)\right|=\left|\left(\mathcal{K}^{-1} \mathcal{M} \mathbf{f}, \mathcal{K} \mathcal{M}^{-1} \mathbf{w}\right)\right|_{L^{2}\left(\Omega ; \mathbb{R}^{2}\right)} \leq\left. C|| \mathcal{K}^{-1} \mathcal{L}^{*} \mathbf{w}\right|_{L^{2}\left(\Omega ; \mathbb{R}^{2}\right)} .
$$

We conclude that $J_{f}$ is bounded on the subspace of $\mathcal{H}_{\mathcal{K}^{-1}}(\Omega)$ consisting of elements having the form $\mathcal{L}^{*} \mathbf{w}$. Extending the operator to a bounded linear functional on the entire space, the Riesz Representation Theorem guarantees the existence of an element $\mathbf{v} \in \mathcal{H}_{\mathcal{K}^{-1}}(\Omega)$ for which

$$
(\mathbf{f}, \mathbf{w})_{L^{2}\left(\Omega ; \mathbb{R}^{2}\right)}=\left(\mathbf{v}, \mathcal{L}^{*} \mathbf{w}\right)_{\mathcal{H}_{\mathcal{K}^{-1}}(\Omega)} .
$$

The proof is completed by taking $\mathbf{u}=-\mathcal{K}^{-2} \mathbf{v}$ and applying (31).

Remark. If the vector $\mathbf{u}$ is sufficiently differentiable, then we can replace $u_{1}$ by $u_{x}$ and $u_{2}$ by $u_{y}$ for a scalar function $u(x, y)$. Formally, we then obtain from Corollary 4 the existence of a weak solution to eq. (1), under mixed boundary conditions consisting of Dirichlet conditions

$$
u_{x} d x+u_{y} d y=0
$$

on $G$ and co-normal conditions

$$
K u_{x} d y-u_{y} d x=0
$$

on the complement of $G$.

However, the existence of a suitable domain $\Omega$ remains to be demonstrated.

It is apparent that the conditions on $\Omega$ are non-vacuous if we consider one of the simplest kinds of piecewise smooth domains, a box with a vertex at the origin. Let $y_{0}$ be a positive number, let $x_{0}=y_{0}^{2}$, and let $\Omega$ be the rectangle formed by the line segments 


$$
\begin{aligned}
& I: 0 \leq x \leq x_{0}, y=y_{0} ; \\
& I I: x=0,0 \leq y \leq y_{0} ; \\
& I I I: 0 \leq x \leq x_{0}, y=0 \\
& I V: x=x_{0}, 0 \leq y \leq y_{0} .
\end{aligned}
$$

On the line segments $I$ and $I I$, both (27) and (28) are satisfied. On the line segments $I I I$ and $I V,(28)$ is satisfied. So we can take $G$ to be a nonempty subset of $I \cup I I$. Or we can take $G$ to be the empty set, in which case Theorem 3 guarantees a weak solution to the conormal problem on $\Omega$. This problem is hard to solve for equations of Tricomi type; see [21] and the comments in Sec. 4 of [13]. In the cold plasma case, it is the weak Dirichlet problem which is hard to solve, as it is not obvious that there is a domain on which $G$ can be taken to be the entire boundary.

While the restriction of the boundary arcs to vertical and horizontal lines obviously simplifies the analysis, it is also clear that the existence argument for the mixed or co-normal problem extends to domains more general than a rectangle. On the elliptic region, all that is required in order for (28) to hold with our choices of $b$ and $c$ is that $d x / d s$ and $d y / d s$ both be nonnegative, where $d s$ is the element of arc length on the boundary. On the hyperbolic region, all that is required for either (27) or (28) to hold is that $d x / d s$ and $d y / d s$ both be non-positive. Thus a suitable domain might have the form of a lens about that segment of the sonic curve $x=y^{2}$ which lies in the first quadrant of the $x y$-plane. It is required that the upper boundary of the lens remain in the hyperbolic region of the equations without violating the condition $d y / d s \leq 0$. For example, let the hyperbolic boundary be given by the curve $y=x^{q}$ over the interval $x \in[0,1]$, for $q \in(0,1 / 2)$; let the elliptic boundary be given by the curve $y=x^{r}$ over the interval $x \in[0,1]$, for $r>1$; and let $G$ be a subset of the hyperbolic boundary.

\section{A SOMEWhat SMOOThER CLASS OF Distribution SOLUTiOnS}

It is also possible to strengthen the result of Sec. 2 in a different direction, by placing hypotheses on $\Omega$ which imply more smoothness on the part of the distribution solution than mere square-integrability.

Following Sec. 2 of [12] we consider a one-parameter family $\psi_{\lambda}(x, y)$ of inhomogeneous dilations given by

$$
\psi_{\lambda}(x, y)=\left(\lambda^{-\alpha} x, \lambda^{-\beta} y\right),
$$

where $\alpha, \beta, \lambda \in \mathbb{R}^{+}$. These determine an associated family of operators

$$
\Psi_{\lambda} u=u \circ \psi_{\lambda} \equiv u_{\lambda}
$$

Denote by $D$ the vector field

$$
D u=\left[\frac{d}{d \lambda} u_{\lambda}\right]_{\mid \lambda=1}=-\alpha x \partial_{x}-\beta y \partial y .
$$

An open set $\Omega \subseteq \mathbb{R}^{2}$ is said to be star-shaped with respect to the flow of $D$ if $\forall\left(x_{0}, y_{0}\right) \in \bar{\Omega}$ and each $t \in[0, \infty]$ we have $F_{t}\left(x_{0}, y_{0}\right) \subset \bar{\Omega}$, where

$$
F_{t}\left(x_{0}, y_{0}\right)=(x(t), y(t))=\left(x_{0} e^{-\alpha t}, y_{0} e^{-\beta t}\right)
$$


If $k$ is a given function on $\Omega$, we define the space $L^{2}(\Omega ;|k|)$ and its dual, consisting, respectively, of functions $u$ for which the norms

and

$$
\|u\|_{L^{2}(\Omega ;|k|)}=\left(\int_{\Omega}|k| u^{2} d x d y\right)^{1 / 2}
$$

$$
\|\left. u\right|_{L^{2}(\Omega ;|k|-1)}=\left(\int_{\Omega}|k|^{-1} u^{2} d x d y\right)^{1 / 2}
$$

are finite; see 13, Sec. 3, for details.

Denote by $v$ a $C^{1}$ solution of the Cauchy problem

$$
H v=u \text { in } \Omega
$$

with $v$ vanishing on $\partial \Omega \backslash\{0,0\}$,

$$
\lim _{(x, y) \rightarrow(0,0)} v(x, y)=0
$$

and

$$
H v=a v+b v_{x}+c v_{y} .
$$

We assume in the sequel that $v$ exists. This assumption is justified whenever the following conditions are met: $\Omega$ is star-shaped with respect to the flow of the vector field $V=-(b, c) ; b=m x$ and $c=\mu y ; \mu$ is a positive constant; $m$ is a step function in $x$, taking only positive values, with a single jump at the point $x=0 ; a$ is a negative constant having sufficiently large magnitude. A proof of the sufficiency of these assumptions can be found in step 1 of the proof of Lemma 3.3, 13.

Theorem 5. Suppose that $x \geq 0$ on $\Omega$ and that $\Omega$ is star-shaped with respect to the flow of the vector field $V=-(b, c)$ for $b=m x$ and $c=\mu y$, where $\mu$ is a positive constant and $m$ exceeds $3 \mu$. Then for every $u \in C_{0}^{\infty}(\Omega)$ there exists a positive constant $C$ for which

$$
\|u\|_{L^{2}(\Omega ;|k|)} \leq C|| L u \|_{H^{-1}(\Omega ; k)},
$$

where $k(y)=y^{2}$ and $L$ satisfies (10) with $\kappa=1$.

Proof. Let $v$ satisfy eqs. (35)-(37) on $\Omega$ for $a=-M$, where $M$ is a positive number satisfying

$$
M=\frac{m-3 \mu}{2}-\delta
$$

for some sufficiently small positive number $\delta$. We have the integral identities

$$
(I u, L u) \equiv(v, L u)=(v, L H v) .
$$

Our choice of the coefficients $a, b$, and $c$ are such that $a_{x}=a_{y}=b_{y}=c_{x}=0$ and all second derivatives also vanish. Substitute into Proposition 12 of the Appendix the quantities $K=x-y^{2}, \kappa_{1}=1, \kappa_{2}=0, b=b(x), c=c(y)$. We have $\omega=0$, 


$$
\begin{gathered}
\alpha=K\left(\frac{c_{y}-b_{x}}{2}-a\right)+\frac{1}{2} b+\frac{1}{2} K_{y} c \\
=\left(\frac{m}{2}-\mu-\delta\right) x+\delta y^{2}, \\
\beta=0,
\end{gathered}
$$

and

$$
\gamma=-a-\frac{c_{y}}{2}+\frac{b_{x}}{2}=M-\frac{\mu-m}{2}=m-2 \mu-\delta .
$$

The boundary integral of Proposition 12 vanishes by the compact support of $u$. We find that if $\delta$ is sufficiently small relative to $m$ and $\mu$, then

$$
(v, L H v) \geq \delta \iint_{\Omega}\left(y^{2} v_{x}^{2}+v_{y}^{2}\right) d x d y .
$$

The upper estimate is immediate. One applies inequality (21) to obtain

$$
(v, L u) \leq\|v\|_{H_{0}^{1}(\Omega ; k)}\|L u\|_{H^{-1}(\Omega ; k)},
$$

from which the desired inequality follows by the continuity of $H$ as a map from $H_{0}^{1}(\Omega ; k)$ into $L^{2}(\Omega ;|k|)$. This completes the proof of Theorem 5 .

Corollary 6. Let $\Omega$ be star-shaped with respect to the flow of the vector field $-V=(m x, \mu y)$, where $m$ and $\mu$ are defined as in Theorem 5. Suppose that $x$ is nonnegative on $\Omega$ and that the origin of coordinates lies on $\partial \Omega$. Then for every $f \in L^{2}\left(\Omega ;|k|^{-1}\right)$ there is a distribution solution $u \in H_{0}^{1}(\Omega ; k)$ to the Dirichlet problem (3), (11) where $k=y^{2}$ and $\kappa=1$.

Proof. The proof mirrors the arguments for the existence of a distribution solution in the proof of Theorem 3.2 in [13, so we will again be brief. Define a linear functional $J_{f}$ by the formula

$$
J_{f}(L \xi)=(f, \xi) .
$$

Using the fact that $L$ is self-adjoint for $\kappa=1$, we estimate

$$
\left|J_{f}(L \xi)\right| \leq C|f|_{L^{2}\left(\Omega ;|k|^{-1}\right)}|| L \xi \|_{H^{-1}(\Omega ; k)} .
$$

Thus $J_{f}$ is a bounded linear functional on the subspace of $H^{-1}(\Omega ; k)$ consisting of elements having the form $L \xi$ with $\xi \in C_{0}^{\infty}(\Omega)$. Extending $J_{f}$ to the entire space, the Riesz Representation Theorem guarantees the existence of an element $u \in H_{0}^{1}(\Omega ; k)$ for which

$$
(u, L \xi)=(f, \xi),
$$

where $\xi \in H_{0}^{1}(\Omega ; k)$. This completes the proof.

Remarks. i) While the solution guaranteed by Corollary 2 is only in $L^{2}$, the solution guaranteed by Corollary 6 has a derivative in a weighted $L^{2}$-space. Thus the solution of Corollary 6 is closer to a conventional weak solution than is the solution of Corollary 2. 
ii) The estimates used to obtain inequality (38) extend in a formal way to the weight function $x-y^{2}$ if, in eq. (10), we take $\kappa<0$ with $|\kappa|$ sufficiently large. Choose

$$
\begin{gathered}
a=\frac{2 \delta}{1-\kappa}\left(\frac{5}{2}-2 \kappa\right)+\delta \kappa \\
b=\frac{4 \delta}{1-\kappa} x+m K
\end{gathered}
$$

and

$$
c=\frac{2 \delta}{1-\kappa} y
$$

for

$$
m=\left\{\begin{array}{cc}
\delta & \text { in } \Omega^{+} \\
-\delta & \text { in } \Omega^{-}
\end{array}\right.
$$

$K=x-y^{2}$, and $\delta>0$. In that case, $\omega=0$;

$$
\begin{aligned}
& \alpha=\left\{\begin{array}{cc}
{[\delta(1+2|\kappa|)] K+4 \delta y^{2}} & \text { in } \Omega^{+} \\
-\delta K+4 \delta y^{2} & \text { in } \Omega^{-}
\end{array} ;\right. \\
& \beta=\frac{1}{2}\left[(1-\kappa) c-b_{y}\right]=\left\{\begin{array}{cc}
2 \delta y & \text { in } \Omega^{+} \\
0 & \text { in } \Omega^{-}
\end{array}\right.
\end{aligned}
$$

and

$$
\gamma=-a-\frac{c_{y}}{2}+\frac{b_{x}}{2}=\delta\left(|\kappa|-4 \pm \frac{1}{2}\right) .
$$

The cross terms satisfy

$$
2 \beta \xi \eta \geq-2 \delta\left(y^{2} \xi^{2}+\eta^{2}\right)
$$

SO

$$
\iint_{\Omega}\left(\alpha \xi^{2}+2 \beta \xi \eta+\gamma \eta^{2}\right) d x d y \geq C \iint_{\Omega}\left(|K| \xi^{2}+\eta^{2}\right) d x d y
$$

provided $|\kappa|$ is sufficiently large. On the boundary between $\Omega^{+}$and $\Omega^{-}, K=0$, so along the cut $b$ is a smooth function of $x$ only. If $\Omega$ is star-shaped with respect to the vector field $V=-(b, c)$, then

$$
(b, c) \cdot \widehat{\mathbf{n}}=0
$$

where $\widehat{\mathbf{n}}=(-d y, d x)$ is the unit normal to $\partial \Omega^{ \pm}$. Thus we can obtain the integral identity of Proposition 12 in this case as well. It is not obvious that the extension is more than formal, as it is not obvious that the vector field $V$ produces a smooth solution of the system (35)-(37). 
4.1. An equation of Keldysh type. As we remarked in Sec. 1, if we allow the resonance curve to be tangent to a flux surface along an entire interval rather than at an isolated point, eq. (11) can be replaced by an equation of the form (4). We add lower-order terms to this equation to obtain

$$
L u=x^{2 k+1} u_{x x}+u_{y y}+c_{1} x^{2 k} u_{x}+c_{2} u=0,
$$

where we take $k \in \mathbb{Z}^{+}$and require that the constants $c_{1}$ and $c_{2}$ satisfy $c_{1}<k+1$ and $c_{2}<0$ with $\left|c_{2}\right|$ sufficiently large. Hypotheses on the magnitudes of lower-order terms are quite common for elliptic-hyperbolic equations, such as (40), which are not of real principle type; see the remarks in [19] and compare the conditions on the lower-order terms in Theorem 4 of [7].

Theorem 7. Let a portion of the line $x=0$ lie in $\Omega$ and let the point $(0,0)$ lie on $\partial \Omega$. Assume that $\Omega$ is star-shaped with respect to the vector field $V=-(b, c)$, where $b=m x$, and $c=\mu y$. Let $\mu$ be a positive constant and let

$$
m= \begin{cases}-a / \ell+\mu / 2 \ell-\delta / \ell & \text { in } \Omega^{+} \\ -a / \ell+\mu / 2 \ell+\delta / \ell & \text { in } \Omega^{-}\end{cases}
$$

for a positive constant $\delta$, where $\ell=k+1-c_{1}$. Let a be a negative constant of sufficiently large magnitude. In particular, let a have sufficiently large magnitude that $m$ is positive. Then for every $w \in C_{0}^{\infty}(\Omega)$ there exists a positive constant $C$ for which

$$
\|w\|_{L^{2}(\Omega ;|K|)} \leq C|| L^{*} w \|_{H^{-1}(\Omega ; K)},
$$

where $K=x^{2 k+1}$ and

$$
\begin{gathered}
L^{*} w=x^{2 k+1} w_{x x}+w_{y y}+ \\
\left(4 k+2-c_{1}\right) x^{2 k} w_{x}+\left[2 k\left(2 k+1-c_{1}\right) x^{2 k-1}+c_{2}\right] w
\end{gathered}
$$

is the formal adjoint of the differential operator $L$ of eq. (40) acting on $w$.

Proof. The proof is only different in its details from that of Theorem 5. In Proposition 12 of the Appendix, take $K=x^{2 k+1}, \kappa_{1}=\left(4 k+2-c_{1}\right) x^{2 k}$ and $\kappa_{2}=2 k\left(2 k+1-c_{1}\right) x^{2 k-1}+c_{2}$. Initially we perform all operations over $\Omega^{+}$and $\Omega^{-}$ individually. On the interior of these sub-domains the coefficients are all smooth. We find that

$$
\omega=M x^{2 k-1}+c_{2}(a-m / 2-\mu / 2),
$$

where $M$ is a constant that depends on $k, a, m$, and $c_{1}$ but not on $c_{2}$. Because $a$ is negative and both $m$ and $\mu$ are positive, $\omega$ is positive provided $c_{2}$ is a negative number having sufficiently large magnitude relative to the quantity $M /|a-m / 2-\mu / 2|$. In addition, Proposition 12 implies that

$$
\begin{aligned}
\alpha & =-K\left(a+2 b_{x}\right)+\frac{3}{2}\left(K_{x} b+K b_{x}\right)+\frac{1}{2} K c_{y}-\kappa_{1} b \\
& =x^{2 k+1}\left[\frac{\mu}{2}-a-\left(k+1-c_{1}\right) m\right]=\delta|x| x^{2 k} ;
\end{aligned}
$$


and

$$
\beta=\frac{1}{2}\left(K_{x}-\kappa_{1}\right) c=\frac{1}{2}\left(c_{1}-2 k-1\right) \mu y x^{2 k} ;
$$

$$
\begin{gathered}
\gamma=-a-\frac{c_{y}}{2}+\frac{b_{x}}{2}= \\
\begin{cases}-(1+1 / 2 \ell) a+(1 / 4 \ell-1 / 2) \mu-\delta / 2 \ell & \text { in } \Omega^{+} \\
-(1+1 / 2 \ell) a+(1 / 4 \ell-1 / 2) \mu+\delta / 2 \ell & \text { in } \Omega^{-}\end{cases}
\end{gathered}
$$

Then $\gamma \geq \delta$ by the hypotheses on the sign and magnitude of $a$. We can write $\beta$ in the form

$$
2 \beta=\left(c_{1}-2 k-1\right) \mu y x^{k+1 / 2} x^{k-1 / 2} .
$$

Then $\forall \varepsilon>0$,

$$
2 \beta \xi \eta \geq-\left|c_{1}-2 k-1\right| \mu|y|\left(\varepsilon|x| x^{2 k} \xi^{2}+\frac{1}{\varepsilon}|x|^{2 k-1} \eta^{2}\right) .
$$

Choose $\varepsilon$ so small that

$$
\left|c_{1}-2 k-1\right| \mu \max _{\bar{\Omega}}|y| \varepsilon<\delta
$$

and $|a|$ so large that

$$
\frac{1}{\varepsilon} \max _{\bar{\Omega}}|x|^{2 k-1}<-\left(1+\frac{1}{2 \ell}\right) a+\left(\frac{1}{4 \ell}-\frac{1}{2}\right) \mu-\frac{\delta}{2 \ell} .
$$

We can do this, as $|\Omega|$ is bounded and $k \geq 1$. Then, arguing as in the proofs of Theorems 1 and 3, we find that

$$
\iint_{\Omega}\left(\alpha \xi^{2}+2 \beta \xi \eta+\gamma \eta^{2}\right) d x d y \geq C \iint_{\Omega}\left(|K| \xi^{2}+\eta^{2}\right) d x d y .
$$

The function $b(x)$ fails to be differentiable on the boundary between $\Omega^{+}$and $\Omega^{-}$. But the coefficients of the boundary terms involving $b_{x}$ either vanish on the line $K=0$ or cancel out, and the remaining boundary terms are smooth. Thus we can integrate over $\Omega^{+}$and $\Omega^{-}$, using the support of $v$ and the Divergence Theorem, and obtain

$$
(v, L H v) \geq C \iint_{\Omega}\left(|K| v_{x}^{2}+v_{y}^{2}\right) d x d y
$$

The remainder of the proof is the same as that of Theorem 5. In particular, the existence of a suitable function $v$ still follows from Lemma 3.3 of [13]: the roles of $x$ and $y$ in that argument are symmetric in the sense that the proof is not affected if the gap in differentiability is shifted from $c(y)$ to $b(x)$.

Corollary 8. Let $\Omega$ and $V$ be defined as in Theorem \%. Then for every $f \in$ $L^{2}\left(\Omega ;|K|^{-1}\right)$ there is a distribution solution $u$ to the Dirichlet problem (40), (11) lying in $H_{0}^{1}(\Omega ; K)$ for $K=x^{2 k+1}$.

Proof. The proof is obtained by arguing exactly as in the proof of Corollary 6 , substituting the different weight class. 
Remark. The weight class of Theorem 7 is more natural than that of Theorem 5 , as we expect any singularities that occur to be localized on the sonic curve $K$. However, we do not obtain uniqueness in any obvious way from either theorem, because we cannot express weak solutions to either eq. (3) or eq. (40) as the limit of an appropriately defined sequence of approximations, as in Sec. 3 of [13]. We discuss this problem further in Sec. 5.1.

\section{INEQUALITIES LEADING TO UNIQUE SOLUTIONS}

In the case of equations of Tricomi type, one can say much more. In fact, Lupo, Morawetz, and Payne are able to show the existence, with a certain degree of regularity, of unique weak solutions to the Dirichlet problem for equations of Tricomi type ([13, Secs. 3 and 5). One problem with adapting that approach to the present case is the geometry of the sonic curve. The simplest approach is to introduce a coordinate transformation which straightens out the sonic curve. One then obtains a problem of Tricomi type, but the coordinate transformation is singular at the origin, which is precisely the point of interest in the problem. In fact, research suggests that the singularity at the origin is an intrinsic property of the model [22, [17, which is not the case, for example, for the Tricomi equation. This leads us to expect that solutions to boundary-value problems for eq. (1) will live in a rougher space than solutions to corresponding problems for eq. (2). In this section we discuss obstructions to obtaining a theorem on the unique existence of solutions to closed boundary-value problems for equations of Keldysh type. We then impose rather harsh conditions in order to obtain the existence of a unique solution to a Dirichlet problem for the cold plasma model.

5.1. On the difficulty of obtaining uniqueness. A simple but generic example will illustrate the difficulty of extending, to equations of Keldysh type, methods developed for proving the weak existence of a unique solution to closed boundaryvalue problems for equations of Tricomi type. In our example the energy estimates used to obtain $H_{0}^{1}(\Omega ; K)$-existence fail for a self-adjoint operator, whereas the convergence arguments used to obtain uniqueness seem to require a self-adjoint operator. Obviously, our example says nothing about whether a unique solution exists, even for this example, but only about the failure of a direct application of methods developed for equations of Tricomi type.

Consider an equation of the form

$$
\left[K(x) u_{x}\right]_{x}+u_{y y}=0
$$

where the smooth function $K(x)$ changes type on the line $x=0$; purely notational alterations $-e . g$., replacing $x$ by $\left(x-x_{0}\right)$ in the definition of $b(x)-$ will extend the argument to change of type on any vertical "flux line" $x=x_{0}$.

In order to guarantee the existence of a smooth solution to the system (35)-(37), we choose $b=m x$ and $c=\mu y$ in that system, where $\mu$ is positive constant and $m$ is a step function which jumps at the line $x=0$ and has the same sign as $\mu$. We take $a$ to be a constant of sufficiently large magnitude having the sign opposite to that of $\mu$ and $m$. The difficulties will not arise from the choices of $\mu$ and $a$, as the argument would be the same for any constants. Moreover, the same difficulties that appear for our choice of vector field will appear if $b=m x$ is replaced by $b=m f(x)$, 
where $f$ is any analytic function vanishing on the flux line (on which $m$ has a jump discontinuity).

The definition of weak solution introduced in 13. for closed elliptic-hyperbolic boundary-value problems is intermediate between the distribution solutions studied in the preceding sections and the strong solutions which we will study in Sec. 5.2. In accordance with that definition, a weak solution to the Dirichlet problem (42), (11) will be a function $u \in H_{0}^{1}(\Omega ; K)$ for which

$$
\langle L u, \xi\rangle=\langle f, \xi\rangle
$$

for every $\xi \in H_{0}^{1}(\Omega ; K)$, where $L$ is the differential operator of (42). Integrating by parts,

$$
\langle L u, \xi\rangle \equiv \iint_{\Omega}\left(\left[K(x) u_{x}\right]_{x}+u_{y y}\right) \xi d x d y=-\iint_{\Omega}\left(K u_{x} \xi_{x}+u_{y} \xi_{y}\right) d x d y .
$$

In this case the existence of a weak solution is equivalent to the existence of a sequence $u_{n} \in C_{0}^{\infty}(\Omega)$ such that

$$
\left\|u_{n}-u\right\|_{H_{0}^{1}(\Omega ; K)} \rightarrow 0 \text { and }\left\|L u_{n}-f\right\|_{H^{-1}(\Omega ; K)} \rightarrow 0
$$

as $n$ tends to infinity. We can thus obtain uniqueness from weak existence by assuming the existence of two solutions and subtracting their approximating sequences.

Arguing as in the proof of Theorem 7 in order to establish the existence of a solution, we estimate the coefficients $\omega, \beta, \alpha$, and $\gamma$. We find that $\omega=\beta=0$, and that

Notice that

$$
\alpha=K(x)\left\{-a+\frac{\mu}{2}+\left[\frac{x K^{\prime}(x)}{K(x)}-1\right] \frac{m}{2}\right\} .
$$

$$
\lim _{x \rightarrow 0} \frac{x K^{\prime}(x)}{K(x)}=\lim _{x \rightarrow 0} \frac{x K^{\prime}(x)}{[K(x)-K(0)]}=1
$$

as $K(0)=0$.

In order for eq. (42) to change type, $K(x)$ must be monotonic in at least a small interval about $x=0$. Initially, suppose that $K^{\prime}(x)$ is positive near $x=0$. The sum $-a+\mu / 2$ cannot be zero, as $a$ and $\mu$ have been given opposite sign in order to insure the existence of a solution to (35)-(37). If $-a+\mu / 2$ is positive, then for small negative values of $x, K(x)$ will be negative. The contribution of $\left[\left(x K^{\prime} / K\right)-1\right] m$ will be small by (43), so $\alpha$ will be negative. If $-a+\mu / 2$ is negative, then $\alpha$ will be negative for small positive values of $x$ for the same reason. An analogous argument pertains to the case in which $K^{\prime}(x)$ is negative near $x=0$.

This example suggests that proving the uniqueness of weak solutions to equations of Keldysh type - and to more complicated non-Tricomi equations - along the lines of [13. will require, at the very least, a quite different choice of vector field $(b, c)$, which will significantly affect the method.

For these reasons we will derive uniqueness from the existence of strong solutions rather than from the linearity of the differential operator on weak solutions. In order to do this, we change the boundary conditions from closed conditions to open conditions. 
5.2. Strong solutions. In the sequel we consider a generalization of the cold plasma model:

$$
L \mathbf{u}=\mathbf{f}
$$

for an unknown vector

$$
\mathbf{u}=\left(u_{1}(x, y), u_{2}(x, y)\right)
$$

and a given vector

$$
\mathbf{f}=\left(f_{1}(x, y), f_{2}(x, y)\right),
$$

where $(x, y) \in \Omega \subset \mathbb{R}^{2}$. Here

$$
(L \mathbf{u})_{1}=[x-\sigma(y)] u_{1 x}+\kappa_{1} u_{1}+\kappa_{2} u_{2 y},
$$

where $\kappa_{1}$ and $\kappa_{2}$ are constants; $\sigma(y) \geq 0$ is a continuously differentiable function of its argument satisfying

$$
\sigma(0)=\sigma^{\prime}(0)=0,
$$

and

$$
\sigma^{\prime}(y)>0 \forall y>0,
$$

In the special case in which $\sigma(y)=y^{2}, \kappa_{2}=0,\left(f_{1}, f_{2}\right)=(f, 0)$, the components of the vector $\mathbf{u}$ are continuously differentiable, and $u_{1}=u_{x}, u_{2}=u_{y}$ for some twice-differentiable function $u(x, y)$, the first-order system (44)-(46) reduces to eq. (3).

We say that a vector $\mathbf{u}=\left(u_{1}, u_{2}\right) \in L^{2}(\Omega)$ is a strong solution of an operator equation of the form (44), with given boundary conditions, if there exists a sequence $\mathbf{u}^{\nu}$ of continuously differentiable vectors, satisfying the boundary conditions, for which $\mathbf{u}^{\nu}$ converges to $\mathbf{u}$ in $L^{2}$ and $L \mathbf{u}^{\nu}$ converges to $\mathbf{f}$ in $L^{2}$.

A sufficient condition for a vector to be a strong solution was formulated by Friedrichs [6] (see also [10]). An operator $L$ associated to an equation of the form

$$
L \mathbf{u}=A^{1} \mathbf{u}_{x}+A^{2} \mathbf{u}_{y}+B \mathbf{u},
$$

where $A^{1}, A^{2}$, and $B$ are matrices, is said to be symmetric-positive if the matrices $A^{1}$ and $A^{2}$ are symmetric and the matrix

$$
Q \equiv 2 B^{*}-A_{x}^{1}-A_{y}^{2}
$$

is positive-definite, where $B^{*}$ is the symmetrization of the matrix $B$ :

$$
B^{*}=\frac{1}{2}\left(B+B^{T}\right) .
$$


If $L$ is not symmetric-positive, then we may consider the equation

$$
E L \mathbf{u}=E \mathbf{f}
$$

for a non-singular matrix $E$ chosen so that $E L$ is symmetric-positive.

Define the matrix

$$
\beta=n_{1} A_{\mid \partial \Omega}^{1}+n_{2} A_{\mid \partial \Omega}^{2},
$$

where $\mathbf{n}=\left(n_{1}, n_{2}\right)$ is the outward-pointing normal vector on $\partial \Omega$. Let $\mathcal{N}(x, y)$, $(x, y) \in \partial \Omega$, be a linear subspace of the vector space $V$, where $\mathbf{u}: \Omega \cup \partial \Omega \rightarrow V$. Suppose that $\mathcal{N}(x, y)$ depends smoothly on $x$ and $y$. The boundary condition that $u$ lie in $\mathcal{N}$ is admissible if $\mathcal{N}$ is a maximal subspace of $V$ and if the quadratic form $(\mathbf{u}, \beta \mathbf{u})$ is non-negative on $\partial \Omega$.

It is sufficient for admissibility that there exist a decomposition

$$
\beta=\beta_{+}+\beta_{-},
$$

for which the following three conditions hold:

i) The direct sum of the null spaces for $\beta_{+}$and $\beta_{-}$spans the restriction of $V$ to the boundary;

ii) the intersection of the ranges of $\beta_{+}$and $\beta_{-}$have only the vector $\mathbf{u}=0$ in common;

iii) the matrix $\mu=\beta_{+}-\beta_{-}$satisfies

$$
\mu^{*}=\frac{\mu+\mu^{T}}{2} \geq 0 .
$$

If these conditions are satisfied, then the boundary condition

$$
\beta_{-} \mathbf{u}=0 \text { on } \partial \Omega
$$

is admissible for eq. (44) and the boundary condition

$$
\mathbf{w}^{T} \beta_{+}^{T}=0 \text { on } \partial \Omega
$$

is admissible for the adjoint problem

$$
L^{*} \mathbf{w}=\mathbf{g} \text { in } \Omega .
$$

Moreover, both problems can be shown to possess unique, strong solutions.

Write the system (44)-(49) in the matrix form

$$
\begin{gathered}
L \mathbf{u}=\left(\begin{array}{cc}
x-\sigma(y) & 0 \\
0 & -1
\end{array}\right)\left(\begin{array}{l}
u_{1} \\
u_{2}
\end{array}\right)_{x}+\left(\begin{array}{ll}
0 & 1 \\
1 & 0
\end{array}\right)\left(\begin{array}{l}
u_{1} \\
u_{2}
\end{array}\right)_{y} \\
+\left(\begin{array}{cc}
\kappa_{1} & \kappa_{2} \\
0 & 0
\end{array}\right)\left(\begin{array}{l}
u_{1} \\
u_{2}
\end{array}\right) .
\end{gathered}
$$

We will show the existence of strong solutions to a subclass of equations for the operator $L$.

Theorem 9. Assume that on the elliptic boundary of $\Omega$

$$
b n_{1}+c n_{2} \geq 0,
$$


where $n_{1}$ and $n_{2}$ are components of the outward-pointing normal vector at each point of $\partial \Omega$, and where $b$ and $c$ satisfy, for $K=x-\sigma(y)$, the inequalities

$$
\begin{gathered}
2 b \kappa_{1}-b_{x} K-b+c_{y} K-c \sigma^{\prime}(y)>0 \text { in } \Omega ; \\
\left(2 b \kappa_{1}-b_{x} K-b+c_{y} K-c \sigma^{\prime}(y)\right)\left(2 c \kappa_{2}+b_{x}-c_{y}\right) \\
-\left(b \kappa_{2}+c \kappa_{1}-c_{x} K-c-b_{y}\right)^{2}>0 \text { in } \Omega ; \\
2 c \kappa_{2}+b_{x}-c_{y}>0 \text { in } \Omega ; \\
b n_{1}-c n_{2} \leq 0 \text { on }(\partial \Omega)^{-} ; \\
c K n_{1}+b n_{2} \geq 0 \text { on }(\partial \Omega)^{-},
\end{gathered}
$$

and

where $(\partial \Omega)^{-}$is the hyperbolic boundary. Then equation (44), with L given by (53) and the Dirichlet condition

$$
-u_{1} n_{2}+u_{2} n_{1}=0
$$

imposed on the elliptic portion of $\partial \Omega$, has a unique, strong solution on $\Omega$ for every $\mathbf{f} \in L^{2}$.

Proof. Define the matrix

$$
E=\left(\begin{array}{cc}
b & -c K \\
c & b
\end{array}\right)
$$

Then the operator $E L$ is symmetric-positive by conditions (55)-(57). In order to show the existence of strong solutions on $\Omega$ it is convenient to produce a decomposition of the matrix

$$
\beta=\left(\begin{array}{cc}
K\left(b n_{1}-c n_{2}\right) & c K n_{1}+b n_{2} \\
c K n_{1}+b n_{2} & -\left(b n_{1}-c n_{2}\right)
\end{array}\right)
$$

On the elliptic boundary, choose

$$
\beta_{+}=\left(\begin{array}{cc}
K b n_{1} & b n_{2} \\
K c n_{1} & c n_{2}
\end{array}\right)
$$

and

$$
\beta_{-}=\left(\begin{array}{cc}
-K c n_{2} & K c n_{1} \\
b n_{2} & -b n_{1}
\end{array}\right) .
$$

Then $\beta_{-} u=0$ under the boundary condition (60). Moreover, the intersection and range of the two matrices satisfy the conditions for admissibility. We have 


$$
\mu^{*}=\left(b n_{1}+c n_{2}\right)\left(\begin{array}{cc}
K & 0 \\
0 & 1
\end{array}\right),
$$

so condition (54) implies that Dirichlet conditions (60) are admissible on the elliptic part of the boundary.

On the hyperbolic boundary we choose $\beta=\beta_{+}$and choose $\beta_{-}$to be the zero matrix. Then the matrix $\mu^{*}$ is nonnegative on $(\partial \Omega)^{-}$by assumptions (58) and (59). Because the other conditions for admissibility are satisfied trivially on $(\partial \Omega)^{-}$, the proof of Theorem 9 is complete.

Remarks. i) In order to show that inequalities (55)-(159) are non-vacuous, we let $\sigma(y)=y^{2}, \kappa_{1}=\kappa_{2}=0, b=M+N K / 2$, and $c=N y$, where $M$ and $N$ are negative constants, $|M|$ is sufficiently large, and $\Omega \subset \mathbb{R} \times \mathbb{R} \backslash \mathbb{R}^{-}$. Then (55)-(57) are satisfied. Moreover, inequalities (58), (59) will be satisfied in a canonical basis $\left(n_{1}, n_{2}\right)=(-d y, d x)$ provided $d y / d s$ and $d x / d s$ are both nonpositive. This suggests that, under the canonical choice of basis, the hyperbolic boundary in Theorem 9 could be a sufficiently thin lens in the first quadrant, the lower boundary of the lens lying along the sonic curve. As a particularly simple example, let the hyperbolic boundary be the arc of the circle

$$
(x-1)^{2}+y^{2}=1
$$

connecting the points $(0,0)$ and $(1,1)$. If $|M|$ is sufficiently large, then (54) requires only that $d y / d s$ be bounded below away from zero on the elliptic boundary.

ii) Under the same choice of basis, in the special case $u_{1}=u_{x}, u_{2}=u_{y}$, we recover condition (32) from condition (60) and condition (33) from the adjoint condition $K u_{1} n_{1}+u_{2} n_{2}=0$.

iii) A hidden smoothness assumption is contained in the choice of the component $f_{2}$ to be zero in eq. (44), as that would imply, in the case $u_{1}=u_{x}, u_{2}=u_{y}$, the equivalence of mixed partial derivatives of the solution. Presumably such a condition would be violated at the origin, at which point the difference of the mixed partial derivatives might be a delta function. If the difference were somewhat smoother than a delta function - that is, an $L^{2}$ function, then the methods of this section could be applied.

iv) There is a geometric analogy for condition (54): Consider a domain which is star-shaped with respect to the flow of a given vector field $D$ satisfying (34). Then the boundary will be starlike with respect to $D$ in the sense that $\alpha n_{1}+\beta n_{2} \geq 0$, or, in terms of the basis used in remarks $i$ ) and ii), $\beta d x-\alpha d y \geq 0$, on the boundary (c.f. [12]). We have avoided imposing the hypothesis that $\Omega$ is $D$-star-shaped in Theorems 3 and 9 , although it would have been possible to do so formally. The reason is that equations of the form (3) are only interesting if the origin is included in the domain, whereas condition $b^{2}+K c^{2}>0$ of Theorem 3 and (55) of Theorem 9 are problematic if $b$ and $c$ are homogeneous functions passing through the origin.

\section{WEAK SOLUTIONS IN $L^{2}$}

The fact that solutions to the closed boundary-value problems in Secs. 3 and 4 lie in spaces in which a weight function vanishes at the origin is a strong restriction on their generality. Theorem 9, with the examples given in the remarks following it, 
demonstrates the existence of strong solutions which lie in $L^{2}$, even at the origin; but unfortunately, the boundary conditions in that theorem are open. In this section we show that the existence in $L^{2}$ of weak solutions to open boundary-value problems is easy to obtain for a wide class of boundaries by arguments which are similar to those of $[18$.

Define $G$ to be a subset of the non-characteristic portion of the boundary, $\partial \Omega \backslash \Gamma$, where $\Gamma$ denotes the part of the boundary consisting of characteristic lines. Denote by $W(\Omega)$ the linear space of continuously differentiable functions $\left(w_{1}, w_{2}\right)$ on $\Omega$, satisfying $w_{1}=0$ on $G, w_{2}=0$ on $\partial \Omega \backslash\{\Gamma \cup G\}$,

$$
w_{1} d x+w_{2} d y=0 \forall(x, y) \in \Gamma,
$$

and

$$
\begin{gathered}
\left(L^{*} \mathbf{w}\right)_{1}=[x-\sigma(y)] w_{1 x}+\left(1-\kappa_{1}\right) w_{1}+w_{2 y}, \\
\left(L^{*} \mathbf{w}\right)_{2}=w_{1 y}-w_{2 x}
\end{gathered}
$$

in $\Omega$.

We define a weak solution to eqs. (44)-(46) with $\kappa_{2}=0$, under the mixed boundary conditions

$$
\begin{gathered}
u_{1} d x+u_{2} d y=0 \forall(x, y) \in G, \\
{[x-\sigma(y)] u_{1} d y-u_{2} d x=0 \forall(x, y) \in \partial \Omega \backslash\{\Gamma \cup G\}}
\end{gathered}
$$

to be any $\mathbf{u} \in L^{2}(\Omega)$ such that $\forall \mathbf{w} \in W(\Omega)$,

$$
(\mathbf{w}, \mathbf{f})=-\left(L^{*} \mathbf{w}, \mathbf{u}\right)
$$

under the $L^{2}$ inner product $($,$) .$

Theorem 10. Let the noncharacteristic boundary of $\Omega$ satisfy the differential inequality

$$
\frac{d y}{d x} \geq \frac{-t y}{m+x}
$$

for a sufficiently large positive constant $m$ and a constant $t$ exceeding 1. Take the curve $G$ to be the elliptic boundary of $\Omega$. Let the constant $\kappa_{1}$ in eq. (45) exceed $1 / 2$ and let $\kappa_{2}=0$. Then $\forall \mathbf{w} \in W(\Omega)$ there exists a positive constant $C$ for which

$$
\|\mathbf{w}\|_{L^{2}(\Omega)} \leq C\left\|L^{*} \mathbf{w}\right\|_{L^{2}(\Omega)} .
$$

Proof. Define the functions $b=-(m+x)$ and $c=-t y$. We will place various conditions on $m$, all of which require that it be sufficiently large in comparison with other parameters $-\kappa_{1}, t,|\Omega|,|\sigma|_{\max (\Omega)}$ and $\left|\sigma^{\prime}\right|_{\max (\Omega)}$ - as well as with certain explicit combinations of these parameters. By the continuity of $\sigma$, we can choose $m$ so large that the matrix

$$
M=\left[\begin{array}{cc}
b & c \\
-K c & b
\end{array}\right]
$$


is non-singular on $\Omega$, where $K=x-\sigma(y)$. We have

$$
\left(L^{*} \mathbf{w}, M \mathbf{w}\right)=\iint_{\Omega} Q d x d y+\iint_{\Omega} S d x d y,
$$

where

$$
Q=\alpha w_{1}^{2}+2 \beta w_{1} w_{2}+\gamma w_{2}^{2}
$$

In particular,

$$
\begin{gathered}
\alpha=\frac{1}{2} K\left(c_{y}-b_{x}\right)+\left(\frac{1}{2}-\kappa_{1}\right) b-\frac{1}{2} \sigma^{\prime}(y) c \\
=\left(\kappa_{1}-\frac{t}{2}\right) x+\frac{t-1}{2} \sigma(y)+m\left(\kappa_{1}-\frac{1}{2}\right)+\frac{t y}{2} \sigma^{\prime}(y) .
\end{gathered}
$$

We can choose $m$ so large that $\alpha$ is bounded below away from zero on $\Omega$. Also,

and $\forall \varepsilon>0$,

$$
\begin{gathered}
\gamma=-\frac{1}{2}\left(c_{y}-b_{x}\right)=\frac{t-1}{2}, \\
\beta=-\frac{1}{2}\left(K c_{x}+b_{y}+\kappa_{1} c\right)=\frac{\kappa_{1} t y}{2},
\end{gathered}
$$

$$
2 \beta \omega_{1} \omega_{2} \geq-\left|\kappa_{1} t y\right|\left(\frac{\omega_{1}^{2}}{\varepsilon}+\varepsilon \omega_{2}^{2}\right) .
$$

Choose $\varepsilon$ to be so small that $\left|\kappa_{1} t y\right| \varepsilon<(t-1) / 2$ and $m$ so large that $m\left[\kappa_{1}-(1 / 2)\right]>$ $\left|\kappa_{1} t y\right| / \varepsilon$. Then there is a positive constant $C$ for which

$$
\iint_{\Omega} Q d x d y \geq C \iint_{\Omega}\left(\omega_{1}^{2}+\omega_{2}^{2}\right) d x d y .
$$

Applying the Divergence Theorem, we obtain

$$
\begin{gathered}
\iint_{\Omega} S d x d y= \\
\iint_{\Omega}\left[(-K)\left(t y w_{1} w_{2}+\frac{m+x}{2} w_{1}^{2}\right)+\frac{m+x}{2} w_{2}^{2}\right]_{x} d x d y- \\
\iint_{\Omega}\left[\frac{1}{2}(-K) t y w_{1}^{2}+(m+x) w_{1} w_{2}+\frac{t y}{2} w_{2}^{2}\right]_{y} d x d y= \\
\int_{\partial \Omega}\left[(-K)\left(t y w_{1} w_{2}+\frac{m+x}{2} w_{1}^{2}\right)+\frac{m+x}{2} w_{2}^{2}\right] d y+ \\
\int_{\partial \Omega}\left[\frac{1}{2}(-K) t y w_{1}^{2}+(m+x) w_{1} w_{2}+\frac{t y}{2} w_{2}^{2}\right] d x
\end{gathered}
$$

It is not excluded that the hyperbolic boundary may include one or more characteristic lines $\Gamma$. Repeatedly applying (62) to the terms in $w_{1} w_{2}$ on the boundary integral over $\Gamma$, we obtain

$$
\frac{1}{2} \int_{\Gamma}\left(K w_{1}^{2}+w_{2}^{2}\right)[t y d x-(m+x) d y]=0,
$$

where on the right we have again used (62), and also the characteristic equations

$$
\frac{d x}{d y}= \pm \sqrt{-K}
$$


c.f. [18, (3.24)-(3.26).

On $G, w_{1}=0$ and the boundary integral reduces to

$$
\frac{1}{2} \int_{G} w_{2}^{2}[t y d x+(m+x) d y] .
$$

On $\partial \Omega \backslash\{G \cup \Gamma\}, w_{2}=0$ and the boundary integral reduces to

$$
\frac{1}{2} \int_{\partial \Omega \backslash\{G \cup \Gamma\}} w_{1}^{2}(-K)[t y d x+(m+x) d y] .
$$

Both integrals are nonnegative by (65).

We have shown that

$$
\left(L^{*} \mathbf{w}, M \mathbf{w}\right) \geq C\|\mathbf{w}\|_{L^{2}}^{2}
$$

for some positive number $C$. Because the elements of $M$ are bounded on $\Omega$, applying the Schwarz inequality to the inner product $\left(L^{*} \mathbf{w}, M \mathbf{w}\right)$ yields for all $\mathbf{w} \in W$ and some new constant $C>0$ inequality (66).

Corollary 11. Under the hypotheses of Theorem 10, for every $\mathbf{f} \in L^{2}(\Omega)$ there exists on $\Omega$ a weak solution to the mixed boundary-value problem (44)-(46), (63), 64).

Proof. Apply the Riesz Representation Theorem as in [15] (c.f. [18]).

Remarks. i) This class of boundaries suggests the ice-cream cone-shaped Tricomi domains (see, e.g., Sec. 2 of [11]), rotated by $90^{\circ}$ in the clockwise direction (so that the ice-cream cone is lying on its side, with the cone formed by the intersecting characteristic lines in the second and third quadrants). This rotation is expected, given the similarity of eq. (11) to the Cinquini-Cibrario equation, in which the sonic curve is rotated $90^{\circ}$ with respect to the sonic curve for the Tricomi equation (2). In fact, the sonic curve of (1) is approximated near the origin by the sonic curve of the Cinquini-Cibrario equation.

ii) More generally, Theorem 10 and Corollary 11 remain true for any choice of $M$ for which

$$
\frac{d y}{d x} \geq-\frac{c}{b}
$$

on the characteristic boundary, $\alpha$ and $\gamma$ are bounded below by a positive constant, and $\alpha \gamma-\beta^{2}$ is nonnegative.

iii) In the corresponding theorem of [18, the origin of coordinates was forced to lie on the boundary of the domain. The question of whether weak solutions to boundary-value problems for eqs. (44)-(46) can be shown to exist for cases in which the origin is allowed to be an interior point was raised in Ch. 3 of [25]. In allowing the origin to lie at either a boundary point or an interior point, we have shown the answer to that question to be "yes." 


\section{Appendix. A multiplier identity}

The proofs of Theorems 5 and 7 are based on a fundamental multiplier identity for the composition of a second-order operator and a first-order operator. The proof of the identity is elementary, as it is based on integration by parts followed by an application of the Divergence Theorem. But due to its importance, we provide a full derivation (in slightly greater generality than we need).

Define the operator $L$ on functions $v \in C^{3}(\Omega)$ with $v \equiv 0$ on $\partial \Omega$, by

$$
L v=K(x, y) v_{x x}+v_{y y}+\kappa_{1} v_{x}+\kappa_{2} v,
$$

where the type-change function $K$ and the lower-order coefficients $\kappa_{1}=\kappa_{1}(x, y)$, $\kappa_{2}=\kappa_{2}(x, y)$ are all $C^{3}$ functions. Define

$$
H v=a v+b v_{x}+c v_{y},
$$

where $a$ is a constant; $c$ is a linear function of $x$ and $y$; $b$ is linear in $x$ but possibly nonlinear in $y ; b$ and $c$ have vanishing mixed partial derivatives.

\section{Proposition 12.}

$$
\begin{gathered}
\iint_{\Omega} v \cdot L H v d x d y=\frac{1}{2} \oint_{\partial \Omega}\left(K v_{x}^{2}+v_{y}^{2}\right)(c d x-b d y) \\
+\iint_{\Omega} \omega v^{2}+\alpha v_{x}^{2}+2 \beta v_{x} v_{y}+\gamma v_{y}^{2} d x d y
\end{gathered}
$$

where

$$
\begin{gathered}
2 \omega=\left(K_{x x}-\kappa_{1 x}+2 \kappa_{2}\right) a-\left[\left(K_{x x}-\kappa_{1 x}+\kappa_{2}\right) b\right]_{x} \\
-\left[\left(K_{x x}-\kappa_{1 x}+\kappa_{2}\right) c\right]_{y} ; \\
\alpha=\left(\frac{c_{y}-b_{x}}{2}-a\right) K+\left(\frac{3}{2} K_{x}-\kappa_{1}\right) b+\frac{c}{2} K_{y} ; \\
2 \beta=\left(K_{x}-\kappa_{1}\right) c-\left(c_{x} K+b_{y}\right) ; \\
\gamma=\frac{b_{x}-c_{y}}{2}-a .
\end{gathered}
$$

Proof. Writing

$$
\begin{gathered}
L H v=K\left[\left(a+2 b_{x}\right) v_{x x}+b v_{x x x}+2 c_{x} v_{y x}+c v_{y x x}\right] \\
+\left(a+2 c_{y}\right) v_{y y}+b_{y y} v_{x}+2 b_{y} v_{x y}+b v_{x y y}+c v_{y y y} \\
+\kappa_{1}\left[\left(a+b_{x}\right) v_{x}+b v_{x x}+c_{x} v_{y}+c v_{y x}\right]+\kappa_{2}\left(a v+b v_{x}+c v_{y}\right),
\end{gathered}
$$

we have

$$
v \cdot L H v=\sum_{i=1}^{16} \tau_{i}
$$


where

$$
\begin{aligned}
& \tau_{1}=v K\left(a+2 b_{x}\right) v_{x x}=\left\{\left[\left(a+2 b_{x}\right)\left(K v_{x}-\frac{1}{2} K_{x} v\right)\right] v\right\}_{x} \\
& -K\left(a+2 b_{x}\right) v_{x}^{2}+\frac{1}{2} K_{x x}\left(a+2 b_{x}\right) v^{2} \\
& \tau_{2}=v K b v_{x x x}= \\
& \left\{-\frac{1}{2} K b v_{x}^{2}+\left[b\left(K v_{x x}-K_{x} v_{x}+\frac{1}{2} K_{x x} v\right)+b_{x}\left(K_{x} v-K v_{x}\right)\right] v\right\}_{x} \\
& -\frac{1}{2}\left(K_{x x x} b+3 K_{x x} b_{x}\right) v^{2}+\frac{3}{2}(K b)_{x} v_{x}^{2} \\
& \tau_{3}=2 v K c_{x} v_{y x}=2\left(v K c_{x} v_{y}\right)_{x}-\left(K_{x} c_{x} v^{2}\right)_{y}-2 K c_{x} v_{x} v_{y}+K_{x y} c_{x} v^{2} \\
& \tau_{4}=v K c v_{y x x}= \\
& \left\{v\left[c\left(K v_{y x}-K_{x} v_{y}\right)-K c_{x} v_{y}\right]\right\}_{x}-\left\{\frac{1}{2} K c v_{x}^{2}-\left(\frac{1}{2} K_{x x} c+K_{x} c_{x}\right) v^{2}\right\}_{y} \\
& -\frac{1}{2}\left\{\left[K_{x x y} c+K_{x x} c_{y}+2 K_{x y} c_{x}\right] v^{2}-(K c)_{y} v_{x}^{2}\right\}+(K c)_{x} v_{x} v_{y} \text {; } \\
& \tau_{5}=v\left(a+2 c_{y}\right) v_{y y}=\left[v\left(a+2 c_{y}\right) v_{y}\right]_{y}-\left(a+2 c_{y}\right) v_{y}^{2} ; \\
& \tau_{6}=v b_{y y} v_{x}=\frac{1}{2}\left(b_{y y} v^{2}\right)_{x} \\
& \tau_{7}=2 v b_{y} v_{x y}=\left(2 v b_{y} v_{x}\right)_{y}-2 b_{y} v_{x} v_{y}-\left(b_{y y} v^{2}\right)_{x} ; \\
& \tau_{8}=v b v_{x y y}=-\left(\frac{1}{2} b v_{y}^{2}\right)_{x}+\left[\left(b v_{x y}-b_{y} v_{x}\right) v\right]_{y}+\frac{1}{2}\left[b_{x} v_{y}^{2}+\left(b_{y y} v^{2}\right)_{x}\right]+b_{y} v_{x} v_{y} \\
& \tau_{9}=v c v_{y y y}=-\frac{1}{2}\left(c v_{y}^{2}\right)_{y}+\left[\left(c v_{y y}-c_{y} v_{y}\right) v\right]_{y}+\frac{3}{2} c_{y} v_{y}^{2} \\
& \tau_{10}=v \kappa_{1}\left(a+b_{x}\right) v_{x}=\frac{1}{2}\left[\left(a+b_{x}\right) \kappa_{1} v^{2}\right]_{x}-\frac{1}{2} \kappa_{1 x}\left(a+b_{x}\right) v^{2} ; \\
& \tau_{11}=v \kappa_{1} b v_{x x}= \\
& \left\{\left[\kappa_{1} b v_{x}-\frac{1}{2}\left(\kappa_{1} b\right)_{x} v\right] v\right\}_{x}+\frac{1}{2}\left(\kappa_{1 x x} b+2 \kappa_{1 x} b_{x}\right) v^{2}-\kappa_{1} b v_{x}^{2} \\
& \tau_{12}=v \kappa_{1} c_{x} v_{y}=\frac{1}{2}\left[\left(\kappa_{1} c_{x} v^{2}\right)_{y}-\kappa_{1 y} c_{x}\right] v^{2} \\
& \tau_{13}=v \kappa_{1} c v_{y x}=\left(v \kappa_{1} c v_{y}\right)_{x}-\frac{1}{2}\left[\left(\kappa_{1} c\right)_{x} v^{2}\right]_{y}
\end{aligned}
$$




$$
\begin{gathered}
-\kappa_{1} c v_{x} v_{y}+\frac{1}{2}\left[\kappa_{1 y} c_{x}+\left(\kappa_{1 x} c\right)_{y}\right] v^{2} ; \\
\tau_{14}=\kappa_{2} a v^{2} ; \\
\tau_{15}=v \kappa_{2} b v_{x}=\frac{1}{2}\left(\kappa_{2} b v^{2}\right)_{x}-\frac{1}{2}\left(\kappa_{2} b\right)_{x} v^{2} ; \\
\tau_{16}=v \kappa_{2} c v_{y}=\frac{1}{2}\left(c \kappa_{2} v^{2}\right)_{y}-\frac{1}{2}\left(c \kappa_{2}\right)_{y} v^{2} .
\end{gathered}
$$

Collect terms and integrate over $\Omega$. Applying the Divergence Theorem, taking into account that $v$ (but not necessarily $v_{x}$ or $v_{y}$ ) vanishes on $\partial \Omega$, completes the proof.

Similar estimates are applied, in the proof of Theorem 1, to a product having the simpler form $(H v, L v)$. The matrix identities which underlie the proofs of Theorems 3 and 10 are analogous but also simpler, as the differential operators in those cases are first-order and the equations are in reduced form. In Proposition 12 the operator is effectively third-order and there are zeroth-order terms in the equations.

Acknowledgment. I am grateful to Profs. D. Lupo and K. R. Payne for discussion of this problem, and to the referee for extremely helpful comments.

\section{REFERENCES}

[1] Ju. M. Berezanskii, Expansions in Eigenfunctions of Selfadjoint Operators, American Mathematical Society, Providence, 1968.

[2] A. V. Bitsadze, Equations of the Mixed Type, Pergammon, New York, 1964.

[3] M. Cibrario, Alcuni teoremi di esistenza e di unicita per l'equazione $x z_{x x}+z_{y y}=0$, Atti R. Acc. Torino 68 (1932-1933).

[4] S. Čanić and B. Keyfitz, An elliptic problem arising from the unsteady transonic small disturbance equation, J. Differential Equations 125 (1996), no. 2, 548-574.

[5] V. P. Didenko, On the generalized solvability of the Tricomi problem, Ukrain. Math. J. 25 (1973), 10-18.

[6] K. O. Friedrichs, Symmetric positive linear differential equations, Commun. Pure Appl. Math. 11 (1958), 333-418.

[7] C. Gu, On partial differential equations of mixed type in $n$ independent variables, Commun. Pure Appl. Math. 34 (1981), 333-345.

[8] P. D. Lax, On Cauchy's problem for hyperbolic equations and the differentiability of solutions of elliptic equations, Commun. Pure Appl. Math. 8 (1955), 615-633.

[9] O. Laporte and R. G. Fowler, Weber's mixed boundary-value problem in electrodynamics, $J$. Math. Phys. 8 (1967), no. 3, 518-522.

[10] P. D. Lax and R. S. Phillips, Local boundary conditions for dissipative symmetric linear differential operators, Commun. Pure Appl. Math. 13 (1960), 427-455.

[11] D. Lupo and K. R. Payne, A dual variational approach to a class of nonlocal semilinear Tricomi problems, Nonlinear Differential Equations Appl. 6 (1999), no. 3, 247-266.

[12] D. Lupo and K. R. Payne, Critical exponents for semilinear equations of mixed elliptichyperbolic and degenerate types, Commun. Pure Appl. Math. 56 (2003), 403-424.

[13] D. Lupo, C. S. Morawetz, and K. R. Payne, On closed boundary value problems for equations of mixed elliptic-hyperbolic type, to appear in Commun. Pure Appl. Math.

[14] R. Magnanini, and G. Talenti, Approaching a partial differential equation of mixed elliptichyperbolic type, in Ill-posed and Inverse Problems, Yu. E. Anikonov, A. L. Bukhageim, S. I. Kabanikhin and V. G. Romanov, eds., VSP 2002, pp. 263-276.

[15] C. S. Morawetz, A weak solution for a system of equations of elliptic-hyperbolic type, Commun. Pure Appl. Math. 11 (1958), 315-331. 
[16] C. S. Morawetz, The Dirichlet problem for the Tricomi equation, Commun. Pure Appl. Math. 23 (1970), 587-601.

[17] C. S. Morawetz, D. C. Stevens, and H. Weitzner, A numerical experiment on a second-order partial differential equation of mixed type, Commun. Pure Appl. Math. 44 (1991), 1091-1106.

[18] T. H. Otway, A boundary-value problem for cold plasma dynamics, J. Appl. Math. 3 (2003), no. 1, 17-33.

[19] K. R. Payne, Solvability theorems for linear equations of Tricomi type, J. Math. Anal. Appl. 215 (1997), 262-273.

[20] K. R. Payne, Weak well-posedness of the Dirichlet problem for equations of mixed elliptichyperbolic type, Le Matematiche 60 (2005), 267-279.

[21] M. Pilant, The Neumann problem for an equation of Lavrent'ev-Bitsadze type, J. Math. Anal. Appl. 106 (1985), 321-359.

[22] A. D. Piliya and V. I. Fedorov, Singularities of the field of an electromagnetic wave in a cold anisotropic plasma with two-dimensional inhomogeneity, Sov. Phys. JETP 33 (1971), 210-215.

[23] H. Weitzner, "Wave propagation in a plasma based on the cold plasma model," Courant Inst. Math. Sci. Magneto-Fluid Dynamics Div. Report MF-103, August, 1984.

[24] H. Weitzner, Lower hybrid waves in the cold plasma model, Commun. Pure Appl. Math. 38 (1985), 919-932.

[25] Y. Yamamoto, "Existence and uniqueness of a generalized solution for a system of equations of mixed type," Ph.D. Dissertation, Polytechnic University of New York, 1994.

Department of Mathematics, Yeshiva University, $500 \mathrm{~W}$ 185th Street, New York, New York 10033

E-mail address: otway@yu.edu 